Sharif University of Technology
Scientia Iranica
Transactions E: Industrial Engineering
SCIENTIA

\title{
Coordination of traditional and online group-buying channels considering website promotion effort
}

\author{
Y. Wu, D. Li, and T. Li* \\ School of Business, East China University of Science and Technology, Shanghai 200237, China.
}

Received 17 April 2018; received in revised form 30 October 2018; accepted 22 December 2018

\section{KEYWORDS}

Group buying;

Dual channels;

Channel management;

Revenue coordination;

Promotion effort.

\begin{abstract}
The development of modern technology and e-commerce has given rise to the emergence of many new selling channels, among which group buying has managed to attract numerous new customers rapidly because of such features as good discounts and great convenience. Although a good discount scheme creates sales growth for sellers, it also causes loss in their profit margins. Meanwhile, the business model of group-buying websites is not thoroughly explored in literature. Based on a Stackelberg game framework, this paper studies the equilibrium between a group-buying website and a seller. The optimal pricing and channel decisions of the seller and the optimal group-buying pricing and promotion effort decisions for the website were investigated to offer guidance for their businesses. It was found that the total profit of the whole system could be hurt when the agreement price or revenue-sharing contracts were adopted by the firms. A revenue-cost sharing contract was proposed that could coordinate the total profit. Finally, this study shows how the scale of the seller and the website and the unit cost can affect optimal decisions in equilibrium.
\end{abstract}

(C) 2020 Sharif University of Technology. All rights reserved.

\section{Introduction}

Many companies have been selling their products or services to their consumers through Group-Buying Websites (GBWs) since the early 21st century. On the GBWs, consumers are encouraged to buy together whereby products or services are offered with price discounts. This practice has become popular in a variety of businesses, ranging from catering to entertainment services. With price discounts, GBWs can attract a large number of consumers in a relatively short period of time. According to the research report by Dholakia (2012) [1], GBWs can help the seller attract new consumers (close to $80 \%$ ) even after running group-

*. Corresponding author. Tel.: +862164252899 E-mail addresses: yifanwu@ecust.edu.cn (Y. Wu); 7r7137r521@qq.com (D. Li); litian@ecust.edu.cn (T.Li)

doi: 10.24200 /sci. 2018.50750 .1850 buying activities several times. Thus, for a seller, a GBW may help boost its brand awareness, increase its sales, and expand its markets.

A GBW serves as an additional sales channel for sellers, with promising potential benefits; however, in practice, not all sellers benefit from the GBW channel. Dholakia (2011a) [2] conducted a survey of 324 sellers who use GBWs (e.g., Groupon, LivingSocial, Open Table, Travelzoo, and Buywithme). It was shown that only $55.5 \%$ of the sellers made profits, $26.6 \%$ suffered losses, and $17.9 \%$ broke even. Interestingly, in spite of the popularity of group-buying activities and behaviors, GBWs are not always making money, either. A survey released in China showed that the number of GBWs reached almost 6000 in 2011. However, this number reduced to 123 by the middle of the year 2014, with an average reduction rate of 5.9 websites per day. The international giant GBWs are also experiencing a tough time. In December 2015, Groupon, the world's largest GBW, announced its exit from the Nordic 
market after months of business falling. It was reported that its market value shrunk by $86 \%$ untill 2015 .

In view of the potential benefits brought about by the GBWs, why do the sellers suffer losses? Given the popularity of group-buying behaviors in consumer markets, why the GBWs fail to make money? Under what conditions should a seller add the GBW channel, and if yes, by what contracts?

These research questions are investigated based on the game theoretic approach. A seller provides products or services. It has a traditional offline channel, say retail stores, selling directly to end consumers. It can choose to add a GBW channel. Through the GBW channel, the seller offers its products or services to GBW according to a certain contract, and GBW further sells them to end consumers at a discounted price (a price lower than the traditional channel). The GBW channel has two effects on the seller's market demand. On the one hand, the GBW can bring new consumers to the seller, possibly through sales efforts. On the other hand, because of the discounted price, the GBW cannibalizes the traditional channel by attracting consumers in the traditional channel to the GBW channel. Four types of contracts are considered here: an agreement price contract, a twopart tariff contract, a revenue-sharing contract, and a revenue-cost sharing contract. For each type of contract, we fully characterize the firms' pricing and sales efforts decisions and analyze the seller's decision on the employment of the GBW channel.

The purpose of the study is two-fold. First, we try to generate insights into a seller's strategic decision on whether or not to run the group-buying promotion (with costly efforts). Second, we propose a contracting mechanism between the seller and the GBW website firm so that the traditional channel and the groupbuying channel can be coordinated with the total profit reaching its maximum.

The remainder of the paper is organized as follows. Section 2 reviews the literature. Section 3 introduces the model in the centralized scenario. Section 4 describes the model setting under agreement price contract. Section 5 studies the conditions under which the seller and the website can be coordinated with two-part tariff contract, revenue-sharing contract, or a revenue-cost sharing contract. Section 6 extends our model to investigate the impact of product unit cost and seller's offline consumer size. Concluding remarks are provided in Section 7. All the proofs appear in the appendix.

\section{Literature review}

Information technology has shown important and growing positive impact on business performance [3]. Emerging technologies have spawn myriad applications that have the potential to impact experienced market dramatically [4], and group-buying, a dynamic consumer collective activity [5], is one of such technologies. A large volume of literature has been given to the study of the group-buying mechanism; herein, the literature has been categorized into three dimensions: the profit mechanism of the sellers in group buying, the profit model of the GBWs, and the interplay between the websites and the sellers.

Group buying takes advantage of group cohesion to benefit both consumers and participating sellers, as it can significantly increase sales volumes. Early research on group buying deems it as an online auction activity and focuses on the dynamic pricing mechanisms regarding the quantity and timing of it (e.g., [69]). Such a business model has been replaced by the fixed-price group-buying business model nowadays.

Through a two-year data collection from 2007 to 2009 in the context of urban China, Wang et al. (2013) [5] explored the value that group buying creates for sellers including the cost reduction and brand awareness building, especially for middle-sized and lesser known sellers. Dholakia (2011a) [2], and Dholakia and Tsabar (2011b) [10] held the same opinion that GBWs like Groupon could be an effective marketing tool for startup businesses by studying 150 small businesses that completed promotions on Groupon between June 2009 and August 2010. Heo (2016) [11] discussed how restaurants could utilize group buying as a tool for revenue management. Wu and Zhu (2017) [12] investigated the quality decision for the seller to align with its group-buying strategy when consumers' substitution effect was taken into consideration.

Group buying is also known as voucher discounting in which the sellers sell vouchers through the GBWs and consumers will buy these vouchers and redeem them offline. Edelman et al. (2016) [13] investigated the profitability, pricing discrimination, and advertising effect of discount vouchers. Gao and Chen (2015) [14] found that no show of voucher buyers could be good for (large or start-up) sellers. Ni et al. (2015) [15] studied the optimal pricing strategy of sellers by classifying customers into collectivist and individualistic customers according to actual market information. Taleizadeh et al. (2015) [16] investigated the joint multi discount pricing and ordering problem when demand for deteriorating product changed with time.

Though the group-buying business model provides sellers with a new channel to sell their products, it is doubtful whether it will be profitable for the sellers in the long term due to the extremely deep discounts. With the deep discounts offered to consumers and payouts transferred to the websites (which can range from 20 to $50 \%$ of the revenue), the seller running 
the group-buying promotion can often be left with insufficient revenues to cover its costs [10]. Hence, it is critical for us to explore the benefit when sellers adopt the group-buying channel and to find out how to guarantee the sellers' profits.

For the extant research, the focus is primarily on the sellers' strategies during the group-buying transaction, rather than the websites' strategies. However, as a platform for implementing group-buying, the corresponding websites should receive greater attention. The mechanism of GBW experienced the evolution from dynamic pricing model (e.g., [6-8]) to fixedpricing model (e.g., $[14,15,17])$. The dynamic model focuses on the quantity and pricing scheme, that is, the greater the quantity, the lower the price. However, the business model is too complex for the websites to operate, leading gradually to the cessation of its operation [18]. In 2008, Groupon, originated in Chicago, introduced deal-of-the-day business model, which is the original form of fixed-pricing model [15]. Groupon achieved great success from the second quarter of 2009 to the first quarter of 2011 and has a revenue growth from $\$ 3.3$ million to $\$ 644$ million. The market expanded from 5 North American markets to 175 markets in 43 countries. The number of registered users increased from 152 thousand to 83 million and the number of sellers operating on its platform increased from 212 to 56781. In a short time, many websites began to clone Groupon's business model. By the end of June 2011, China became one of the most competitive group-buying markets in the world [19].

The worldwide rapid expansion of GBWs has prompted much research on the profit mechanism of GBWs. Commission, sometimes called royalty fee that the sellers pay to the websites, is a major revenue source for the website $[13,14,17]$. Zhao et al. (2016) [17] found that the commission charged by the website had a deep influence on sellers' pricing strategies. When the commission is endogenous, one can observe the promotional effect on advertising sellers' service quality. Edelman et al. (2016) [13] promoted a theoretical decision framework for sellers with dual channels, i.e., online and offline channels, that involves how to make a group-buying decision and set an optimal groupbuying price when the deal quantity increases. Unlike other literature focusing on the cost of operating a GBW [15], this study ignores the cost of running groupbuying business in our basic model. Otherwise, we take the effort cost of running group-buying business into consideration and assume that the website's promotion effort level has a linear positive effect on the online demand. This kind of effort cost is quite common in dual-channel supply chains [20].

After several years of rapid development, GBWs face a not so promising future. Take the development of GBWs in China for example; the number of the websites dropped from nearly 6000 in 2011 to 123 in June 2014. Therefore, we should also attach great importance to the survival of GBWs.

Beyond the work on the separate strategies of the sellers and websites, a branch of research has explored the interplay between the seller and the website, among which the cooperation between sellers and the websites is most extensively studied. As mentioned above, commission is a traditional way in which the sellers and the websites use to share total revenue.

Zhao et al. (2016) [17] considered a combination of the transaction-based commission and a fixed payment (a two-part tariff). They provided an effective way to balance the revenue between the seller and the website when there is a deep discount. Similarly, Tran and Desiraju (2017) [21] explored the channel coordination under asymmetric information of manufacturers and the retailers that can provide group buying. They assumed that besides the wholesale price, the retailer would charge a fixed fee for all the products. They found that when the retailer is more informed about the market size than about the level of consumer price sensitivity, the manufacturer will benefit more from group buying. This study also supposes an agreement price combined with a fixed fee when exploring the coordination between the seller and the website in different market scenarios.

Besides the transaction-based commission, more economic methodologies are used to research the interplay between the sellers and the websites. Based on the framework of Stackelberg game, Ni et al. (2015) [22] explored the optimal strategies of the seller and the website when leading the market, respectively. Similar to this, the current study also utilizes the Stackelberg game model to simulate different marketing situations. The difference is that we focus on the market structure including the offline and online consumer size and product cost, while they emphasize consumers' behavior in group buying, that is, the sensibility for the cost to look for people to join group buying together. Bhardwaj and Sajeesh (2017) [23] considered the bargaining power of sellers and the website, and found that when the two retailers compete with each other, the website prefers cooperating with one of them rather than both. Subramanian and Rao (2016) [24] used a traditional revenue sharing model to distribute the total groupbuying revenue. They showed that the cannibalization could be transformed into an advantage by displaying deal sales.

When sellers decide to sell products or services through the group-buying channel, they will not abandon their offline channel, which means they will sell their products/services through the dual channel. Cao et al. (2016) [25] focused on a retailer that sells through multiple distribution channels and studied how an "online-to-store" channel impacted the retailer's 
demand allocations and profitability. Chen et al. (2016) [26] investigated price and quality decisions in dual-channel supply chains, and found that the supply chain performance could be improved due to a new channel augmented. Zhang et al. (2017) [27] investigated the retailer's decisions on channel structure, and concluded that the optimal decision depends on customer acceptance rate of the online channel.

This paper indicates that when deciding to sell through GBW, the seller will make its decision according to the website's platform consumer size. This is indeed the same for the websites, that is to say, they will decide whether to cooperate with each other based on the size of the opposite side. Our work importantly differs from its antecedents in that the prior literature, including the articles discussed above, has considered primarily the website's and the seller's respective optimal decision. In particular, the previous work on group buying does not consider what influences the website's size for seller's optimal marketing decision, which is a primary factor considered in our paper.

\section{Model setting}

\subsection{The seller, the $G B W$, and the market}

A seller provides its product or service to consumers at a constant unit production $\operatorname{cost} c(c \geq 0)$. Two selling channels are available for the seller. Through the traditional offline channel, the seller directly sells to end consumers, say through its retail stores, at a regular price $p_{o}$. Through the online group-buying channel, the seller promotes the product or service through an online Group-Buying Website firm (i.e., a GBW) according to a certain contract; the GBW further sells the product or service to end consumers at a group-buying price $p_{g}$. The seller makes decisions on whether or not to sell through the GBW.

If the seller sells through the single traditional offline channel, we say it runs $O$ model. If the seller chooses to also sell through the GBW, we consider four types of contracts between the seller and the GBW: agreement price, two-part tariff, revenue sharing, and revenue-cost sharing. With respect to the four types of contracts, it can be mentioned that the seller runs $O+G B W$ model, $O+G B W+T$ model, $O+G B W+R$ model, and $O+G B W+R C$ model, respectively.

\subsubsection{O model}

Under the O model, the seller faces consumers in the spot market, with a potential size of $a$. The demand function is given by $q_{o}=a-p_{o}$. If the seller implements the $\mathrm{O}$ model, its profit is given by:

$$
\Pi_{o}=\left(p_{o}-c\right)\left(a-p_{o}\right)
$$

leading to its optimal regular price $p_{o}^{*}=\frac{a+c}{2}$.

\subsection{2. $O+G B W$ model}

Under the $\mathrm{O}+\mathrm{GBW}$ model, the GBW will bring new consumers for the product or service, with a potential size $(b+\gamma)$, at a cost of $\frac{\gamma^{2}}{2}$. Herein, $b$ represents the number of the group-buying product hunters who can actively notice the product once it is advertised by, and available on, the GBW. In addition, the GBW firm can make sales efforts at cost $\frac{\gamma^{2}}{2}$ to attract $\gamma$ more new GBW buyers. For example, the GBW can analyze the purchasing histories and preferences of the consumers in its consumer base to identify the targeted potential consumers for the product.

To eliminate the uninteresting cases, we assume, throughout the paper, that the parameter $b$ is not too large. Mathematically, it is assumed that $b<(a+c) / 2$. In fact, $b$ can be interpreted as the size of consumer base for the product in the GBW channel. The parameter $b$ is usually related to the attractiveness of a GBW. If $b$ is too large, the GBW is too attractive and the demand from the traditional offline channel is negligible.

Assume that the seller's regular price $p_{o}^{*}$ is unchanged when adding the GBW channel. This assumption is consistent with practices. For example, Groupon states that the group-buying sellers' advertised prices must be consistent with the price list of the local business who may be asked to show a written proof of the prices [2]. Subramanian and Rao [24] made a similar assumption by arguing that the seller's regular price would not be affected by a daily deal promotion that is of relatively short duration and offered infrequently.

Under the $\mathrm{O}+\mathrm{GBW}$ model, the GBW cannibalizes the seller's regular sales by transferring a fraction of spot-market consumers from the offline channel to the GBW online channel. The transfer rate is assumed to be $\left(1-\frac{p_{g}}{p_{o}^{*}}\right)$, which relates to the ratio between the group-buying price and the regular price. Clearly, the lower the group-buying price $p_{g}$, the more the spotmarket consumers transfer from the traditional offline channel to the GBW online channel. The market potential in the traditional offline channel becomes $\frac{p_{g}}{p_{o}^{*}} a$ and that in the GBW channel is $\left(1-\frac{p_{g}}{p_{o}^{*}}\right) a+b+\gamma$.

Under the $\mathrm{O}+\mathrm{GBW}$ model, the seller and the GBW firm adopt an agreement price contract. It is the seller who sets the agreement price. With such a contract, the GBW pays an agreement price, $p_{s}\left(c<p_{s}<p_{g}<p_{o}\right)$, to the seller for each deal sold online. The agreement price contract is similar to the wholesale price contract in the supply chain management literature. The $\mathrm{O}+\mathrm{GBW}$ model is displayed in Figure 1.

If the seller implements the $\mathrm{O}+\mathrm{GBW}$ model, its profit is given by:

$$
\Pi_{s}=\left(p_{s}-c\right)\left[\left(1-\frac{p_{g}}{p_{o}}\right) a+b+\gamma-p_{g}\right],
$$




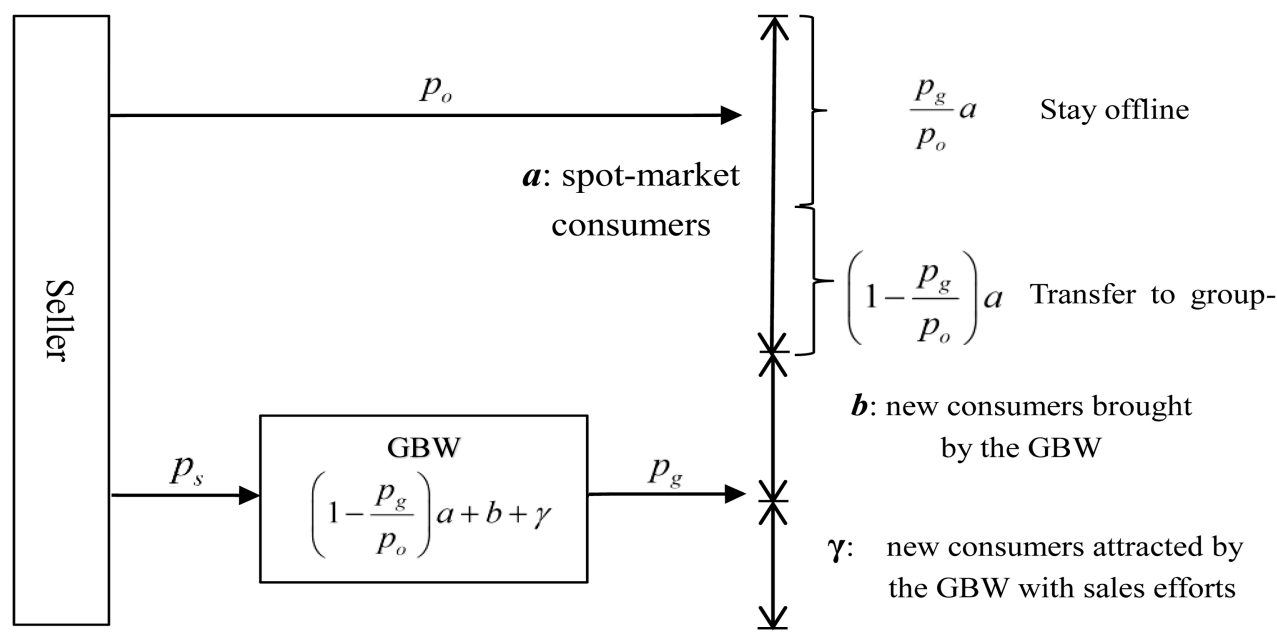

Figure 1. O+GBW model for the seller.

$$
+\left(p_{o}-c\right)\left(\frac{p_{g}}{p_{o}} a-p_{o}\right),
$$

where the first term is the profit obtained from selling to GBW, and the second term is the profit gained from selling to consumers through the traditional offline channel. The GBW's profit is then given by:

$$
\Pi_{g}=\left(p_{g}-p_{s}\right)\left[\left(1-\frac{p_{g}}{p_{o}}\right) a+b+\gamma-p_{g}\right]-\frac{\gamma^{2}}{2} .
$$

From now on, a special case of $a=1$ and $c=0$ will be discussed to simplify the analysis and obtain the key insights. Section 6 extends the current investigation to the impact of spot-market consumer size $a$ and product (service) cost $c$ on the equilibrium and optimal decisions for the seller and the GBW.

\subsection{Benchmark: the centralized setting}

Suppose that the seller and the GBW firm are operated by a central planner. This centralized firm chooses which model to run: the $\mathrm{O}$ model or the $\mathrm{O}+\mathrm{GBW}$ model. In this setting, the seller and the GBW do not need to sign any contract, because the two firms are viewed as a single firm to maximize the overall profits from both the offline and GBW channels.

Under the O model, the centralized firm sells only through its traditional offline channel. Thus, the case is the same as that described in Subsection 3.1.1. The profit of the centralized firm (or the seller) can be written as $\Pi_{o}=p_{o}\left(1-p_{o}\right)$ (Recall that we now focus on the case with $a=1$ and $c=0$ ). The optimal regular price is $p_{o}^{*}=\frac{1}{2}$, and the optimal profit is $\Pi_{o}^{*}=\frac{1}{4}$.

Under the $\mathrm{O}+\mathrm{GBW}$ model, the sales quantities in the GBW channel and in the traditional offline channel are respectively as follows:

$$
q_{g}^{C}= \begin{cases}1-\frac{p_{g}^{C}}{p_{o}^{*}}+b+\gamma-p_{g}^{C}, & p_{g}^{C}<p_{o}^{*} \\ 0, & p_{g}^{C} \geq p_{o}^{*}\end{cases}
$$

and:

$$
q_{s}^{C}= \begin{cases}\frac{p_{g}^{C}}{p_{o}^{*}}-p_{o}^{*}, & p_{g}^{C}<p_{o}^{*} \\ 0, & p_{g}^{C} \geq p_{o}^{*}\end{cases}
$$

where the superscript $c$ denotes the centralized setting and $p_{o}^{*}=\frac{1}{2}$ is the fixed offline regular price. Then, the maximization problem in the centralized firm can be described as follows:

$$
\begin{aligned}
\max _{p_{g}^{C}, \gamma} \Pi^{C} & =p_{g}^{C} \cdot q_{g}^{C}+p_{o}^{*} q_{s}^{C}-\frac{\gamma^{2}}{2}, \\
\text { s.t.: } \quad q_{g}^{C} \geq 0 & \geq \\
q_{s}^{C} & \geq 0 .
\end{aligned}
$$

Solving the maximization problem, we obtain the following lemma:

Lemma 1: In the centralized setting, under the $O+G B W$ model:

1. The optimal group-buying price and sales effort are $p_{g}^{C^{*}}=\frac{2+b}{5}$ and $\gamma^{C^{*}}=\frac{2+b}{5}$, respectively;

2. The optimal profit of the centralized firm is $\Pi^{C^{*}}=$ $\frac{3+8 b+12 b^{2}}{20}$.

The consequent sales quantities through the offline and GBW channels are $q_{s}^{C^{*}}=\frac{3+4 b}{10}$ and $q_{g}^{C^{*}}=$ $\frac{1+3 b}{5}$, respectively. The discount of GBW, defined as the ratio of the group-buying price to the regular price, is $\beta^{C *}=\frac{p_{g}^{C^{*}}}{p_{o}^{*}}=\frac{2(2+b)}{5}$.

When having a greater GBW consumer base (larger $b$ ), the centralized firm adjusts its two instruments. First, when $b$ increases, the centralized firm increases its group-buying price $p_{g}^{C^{*}}$ to raise its unit margin. However, this hurts its sales in the GBW 
channel (note that $q_{g}^{C}$ decreases in $p_{g}^{C}$ ). Second, the centralized firm must make more efforts to attract more new consumers to compensate for the shrinkage of sales caused by a higher $p_{g}^{C^{*}}$. This explains that both $p_{g}^{C}$ and $\gamma^{C^{*}}$ increase in $b$.

Proposition 1: Suppose that the seller and the $G B W$ are centralized to a single firm. The centralized firm adopts the $O+G B W$ model if $b \geq \frac{\sqrt{10}-2}{6}$ and adopts the $O$ model otherwise.

If the seller and the GBW are owned by a single firm, there is no double marginalization problem. The upside of the GBW channel for the centralized firm is that it brings new potential consumers. The downside is that it shifts the spot-market consumers from the offline channel to the GBW channel, which sacrifices the unit profit margin. When the GBW channel is attractive (indicated by large $b$ ), the centralized firm chooses the $\mathrm{O}+\mathrm{GBW}$ model; otherwise, it simply runs the $\mathrm{O}$ model to sell solely on the spot market with a high margin.

\section{Agreement price contract}

In this section, the seller's and the GBW's optimal prices under the agreement price contract are derived, based on which the system performances will be discussed.

The sequence of events is as follows (depicted in Figure 2):

(a) The seller decides whether to add the GBW channel or not;

(b) If the GBW channel is added, then the seller announces its agreement price $p_{s}^{A}$ to the GBW and the GBW decides whether to accept it or not;

(c) If the agreement price contract is agreed, the GBW decides the group-buying price $p_{g}^{A}$ and the sales effort $\gamma^{A}$; the demands and the profits are then realized.

In the seller-leader case, the GBW's decisions depend on the seller's.

\subsection{Optimal decisions under the $O$ model}

Under the O model, the seller's optimal regular price and profit are the same as those in Subsection 3.2. That is, $p_{o}^{*}=\frac{1}{2}$ and $\Pi_{o}^{*}=\frac{1}{4}$.

\subsection{Optimal decisions under the $O+G B W$ model}

Under the O+GBW model, the sales quantities through the online GBW channel and the traditional offline channel are respectively as follows:

$$
q_{g}^{A}= \begin{cases}1-\frac{p_{g}^{A}}{p_{o}^{*}}-p_{g}^{A}+b+\gamma^{A}, & p_{g}^{A}<p_{o}^{*} \\ 0, & p_{g}^{A} \geq p_{o}^{*}\end{cases}
$$

and:

$$
q_{s}^{A}= \begin{cases}\frac{p_{g}^{A}}{p_{o}^{*}}-p_{o}^{*}, & p_{g}^{A}<p_{o}^{*} \\ 0, & p_{g}^{A} \geq p_{o}^{*}\end{cases}
$$

where the superscript $A$ denotes the agreement price contract scenario, and $p_{o}^{*}=\frac{1}{2}$ is the fixed offline regular price. The firms' maximization problems in the agreement price contract scenario can be formulated under $\mathrm{O}+\mathrm{GBW}$ as follows:

$$
\begin{gathered}
\left\{\begin{array}{l}
\max _{p_{s}^{s}} \Pi_{s}^{A}=p_{s}^{A} \cdot q_{g}^{A}+p_{o}^{*} \cdot q_{s}^{A} \\
\max _{p_{g}^{A}, \gamma^{A}} \Pi_{g}^{A}=\left(p_{g}^{A}-p_{s}^{A}\right) \cdot q_{g}^{A}-\frac{\left(\gamma^{s}\right)^{2}}{2}
\end{array}\right. \\
\text { s.t.: } \quad q_{g}^{A} \geq 0, \\
\qquad q_{s}^{A} \geq 0 .
\end{gathered}
$$

This is a dynamic game. A backward induction is used to obtain firms' optimal decisions on prices and sales efforts, as summarized in the following lemma.

Lemma 2: $\quad$ Suppose the seller and the GBW implementing the $O+G B W$ model under the agreement price contract.

1. The optimal agreement price for the seller is $p_{s}^{A *}=$ $\frac{3 b+5}{18}$; the optimal group-buying price and effort for the $G B W$ are $p_{g}^{A^{*}}=\frac{12 b+14}{45}$ and $\gamma^{A^{*}}=\frac{3 b+1}{30}$, respectively;

2. The seller's optimal profit is $\Pi_{s}^{A^{*}}=\frac{9 b^{2}+66 b+16}{180}$; the GBW's optimal profit is $\Pi_{g}^{A^{*}}=\frac{(3 b+1)^{2}}{360}$; the total profit of two firms is $\Pi^{A^{*}}=\Pi_{s}^{A^{*}}+\Pi_{g}^{A^{*}}=$ $\frac{33+138 b+27 b^{2}}{360}$.

The seller's sales quantity through the GBW channel and the traditional offline channel are $q_{g}^{A^{*}}=$ $\frac{3 b+1}{10}$ and $q_{s}^{A^{*}}=\frac{48 b+11}{90}$, respectively. The discount on the GBW is

$$
\beta^{A^{*}}=\frac{p_{g}^{A^{*}}}{p_{o}^{*}}=\frac{2(12 b+14)}{45} .
$$

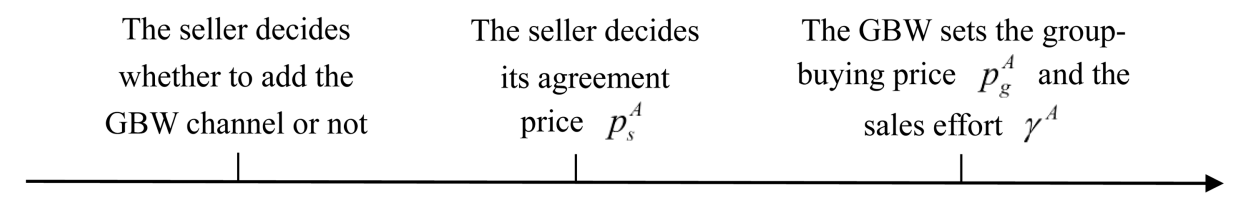

Figure 2. The sequence of events under the Agreement Price Contract. 


\subsection{Whether or not to add the GBW channel} By comparing the seller's profits under the O model and the $\mathrm{O}+\mathrm{GBW}$ model, the following proposition is obtained on the seller's channel model choice.

Proposition 2: Under the agreement price contract scenario, the seller adopts the $O+G B W$ model if $b \geq$ $\frac{5 \sqrt{6}-11}{3}$ and adopts the $O$ model otherwise.

The seller adds the GBW channel if and only if the GBW firm can attract sufficiently more new consumers (i.e., large $b$ ). This is the same as the centralized firm, as shown in Proposition 1.

\section{The pricing coordination of the seller and the GBW}

\subsection{The pricing coordination under the $O+G B W+T$ model}

In this part, the relationship between the seller and the GBW is governed by a two-part tariff (denoted by $\mathrm{T})$ contract, denoted $\left(p_{s}^{T}, F\right)$, whose terms include an agreement price $p_{s}^{T}$ and a fixed fee $F$. Suppose that the website pays the seller a fixed payment and an agreement price for each transaction sold on the website if the seller decides to sell through the online channel.

5.1.1. Optimal decisions under the $O+G B W+T$ model The sequence of events is displayed in Figure 3:

(a) The seller decides whether to add the GBW channel or not:

(b) If yes, the seller announces its agreement price $p_{s}^{T}$ and fixed payment $F$

(c) The GBW decides whether to accept the contract or not and, if yes, decides its group-buying price $p_{g}^{T}$ and the effort $\gamma^{T}$. The demands and the profits are then realized.

The group buying and offline channel demands are:

$$
q_{g}^{T}= \begin{cases}1-\frac{p_{g}^{T}}{p_{o}}+b+\gamma^{T}-p_{g}^{T}, & p_{g}^{T}<p_{o} \\ 0, & p_{g}^{T} \geq p_{o}\end{cases}
$$

and:

$$
q_{s}^{T}= \begin{cases}\frac{p_{g}^{T}}{p_{o}} a-p_{o}, & p_{g}^{T}<p_{o} \\ 0, & p_{g}^{T} \geq p_{o}\end{cases}
$$

The optimization problem is formulated as follows:

$$
\begin{aligned}
& \left\{\begin{array}{l}
\max _{p_{s}^{T}, F^{T}} \Pi_{s}^{T}=p_{s}^{T} \cdot q_{g}^{T}+p_{o} \cdot q_{s}^{T}+F \\
\max _{p_{g}^{T}, \gamma^{T}} \Pi_{g}^{T}=\left(p_{g}^{T}-p_{s}^{T}\right) \cdot q_{g}^{T}-\frac{\left(\gamma^{T}\right)^{2}}{2}-F
\end{array}\right. \\
& \text { s.t.: } \quad q_{g}^{T} \geq 0, \\
& \qquad q_{s}^{T} \geq 0 .
\end{aligned}
$$

Solving the optimal group-buying price and the groupbuying effort, we get $p_{g}^{T^{*}}=\frac{1+b+2 p_{s}^{T}}{5}$ and $\gamma^{T^{*}}=$ $\frac{1+b-3 p_{s}^{T}}{5}$. Then, we can get the offline and online demand as $q_{g}^{T}=\frac{3+3 b-9 p_{s}^{T}}{5}$ and $q_{s}^{T}=\frac{4 b-2+4 p_{s}^{T}}{10}$, respectively. The profit of the seller can be expressed as:

$$
\Pi_{s}^{T^{*}}=\frac{b+3 b p_{s}^{T}-9\left(p_{g}^{T}\right)^{2}}{5}+p_{s}^{T}-\frac{1}{20}+F^{T} .
$$

Solving optimal agreement price and fixed payment regarding seller's profit, we get $p_{s}^{T^{*}}=\frac{3 b+5}{18}$. All the results are summarized below in Lemma 3 .

Lemma 3: When implementing $O+G B W+T$ model:

1. The optimal agreement price and fixed payment for the seller are $p_{s}^{T^{*}}=\frac{3 b+5}{18}$ and $F^{*}=\frac{1+6 b+9 b^{2}}{360}$, respectively. The optimal group-buying price and effort for the $G B W$ are $p_{g}^{T^{*}}=\frac{14+12 b}{45}$ and $\gamma^{T^{*}}=$ $\frac{1+3 b}{30}$, respectively;

2. The seller's optimal profit is $\Pi_{s}^{T^{*}}=\frac{11+46 b+9 b^{2}}{120}$, the $G B W$ 's optimal profit is $\Pi_{g}^{T^{*}}=0$, and the optimal total profit of the two firms is $\Pi^{T^{*}}=\frac{11+46 b+9 b^{2}}{120}$.

The seller's sales quantity through the GBW channel and the traditional offline channel are $q_{g}^{T^{*}}=$ $\frac{1+3 b}{10}$ and $q_{s}^{T^{*}}=\frac{11+48 b}{90}$, respectively. The discount on GBW is $\beta^{T^{*}}=\frac{4(7+6 b)}{45}$.

5.1.2. Whether or not to add the GBW channel By comparing the seller's profits under the O model and the $\mathrm{O}+\mathrm{GBW}+\mathrm{T}$ model, the following proposition on the seller's channel model choice can be obtained.

Proposition 3: The seller adopts the $O+G B W+T$ model if $b \geq \frac{10 \sqrt{7}-23}{9}$ and adopts the $O$ model otherwise.

Proposition 4: Under $O+G B W+T$ model, the fixed payment is increasing with the website's scale, $b$.

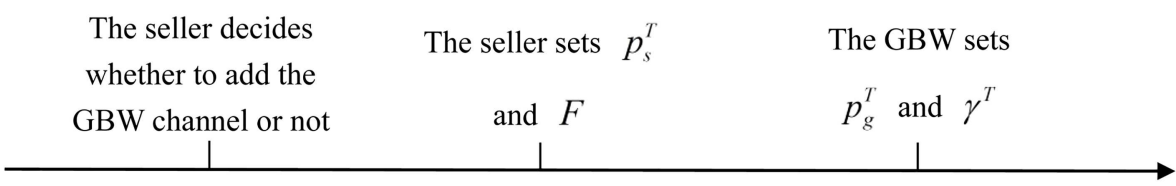

Figure 3. The sequence of events under the $\mathrm{O}+\mathrm{GBW}+\mathrm{T}$ model. 


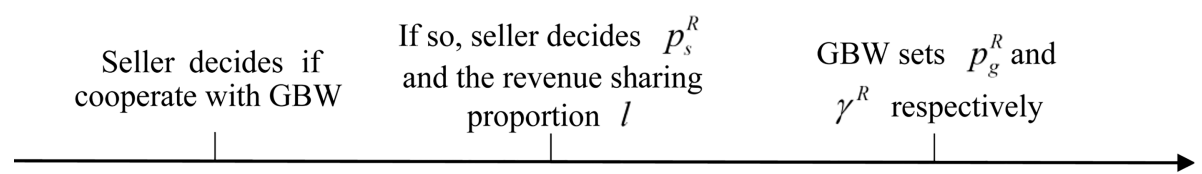

Figure 4. The decision sequence of the seller and Group-Buying Website (GBW) under the $\mathrm{O}+\mathrm{GBW}+\mathrm{R}$ model.

\subsection{The pricing coordination under the $O+G B W+R$ model}

5.2.1. Optimal decisions under the $O+G B W+R$ model

In this part, the seller and the GBW use the revenuesharing contract (denoted by $R$ ), that is, $\left(p_{s}^{R}, l\right)$. The decision sequence is shown in Figure 4.

Under the revenue-sharing contract, the sales quantities in the GBW channel and in the traditional offline channel are respectively as follows:

$$
q_{g}^{R}= \begin{cases}1-\frac{p_{g}^{R}}{p_{o}}+b+\gamma^{R}-p_{g}^{R}, & p_{g}^{R}<p_{o} \\ 0, & p_{g}^{R} \geq p_{o}\end{cases}
$$

and:

$$
q_{s}^{R}= \begin{cases}\frac{p_{g}^{R}}{p_{o}}-p_{o}, & p_{g}^{R}<p_{o} \\ 0, & p_{g}^{R} \geq p_{o}\end{cases}
$$

Firms' maximization problems in the seller-leader case under the revenue-sharing contract can be formulated as follows:

$$
\begin{aligned}
& \left\{\begin{array}{l}
\max _{p_{s}^{R}} \Pi_{s}^{R}=p_{s}^{R} \cdot q_{g}^{R}+p_{o}^{*} \cdot q_{s}^{R} \\
\max _{p_{g}^{R}, \gamma^{R}} \Pi_{g}^{R}=\left(p_{g}^{R}-p_{s}^{R}\right) \cdot q_{g}^{R}-\frac{\left(\gamma^{R}\right)^{2}}{2}
\end{array}\right. \\
& \text { s.t.: } \quad q_{g}^{R} \geq 0, \\
& q_{s}^{R} \geq 0 .
\end{aligned}
$$

All results are summarized in the following lemma.

\section{Lemma 4: Under the $O+G B W+R$ model:}

1. The optimal agreement price and revenue-sharing proportion for the seller are $p_{s}^{R^{*}}=0$ and $l^{*}=$ 1 , respectively; the optimal group-buying price and effort for the $G B W$ are $p_{g}^{R^{*}}=\frac{2+b}{6}$ and $\gamma^{R^{*}}=0$, respectively;

2. The optimal profit for the seller is $\Pi_{s}^{R^{*}}=\frac{b^{2}+4 b+1}{12}$, and the optimal profit for $G B W$ is $\Pi_{g}^{R^{*}}=0$. The total profit of two firms is $\Pi^{R^{*}}=\frac{b^{2}+4 b+1}{12}$.

The seller's sales quantity through the GBW channel and the traditional offline channel are $q_{g}^{R^{*}}=\frac{b}{2}$ and $q_{s}^{R^{*}}=\frac{2 b+1}{6}$, respectively. The discount on the GBW is $\beta^{R^{*}}=\frac{p_{g}^{R^{*}}}{p_{o}^{*}}=\frac{2+b}{3}$.

\subsubsection{Whether or not to add the $G B W$ channel}

By comparing the seller's profits under the O model and the $\mathrm{O}+\mathrm{GBW}+\mathrm{R}$ model, the following proposition on the seller's channel model choice can be obtained.

Proposition 5: The seller adopts the $O+G B W+R$ model if $b \geq \sqrt{6}-2$ and adopts the $O$ model otherwise.

The seller adds the GBW channel if and only if the GBW firm can attract sufficiently more new consumers (i.e., large $b$ ). This is the same as the centralized firm, the $\mathrm{O}+\mathrm{GBW}+\mathrm{T}$ model, and the $\mathrm{O}+\mathrm{GBW}+\mathrm{R}$ model, shown in Propositions 1, 2, and 5. However, a further comparison of the two thresholds of $b$ in Propositions 1 and 3 reveals the following Corollary:

Corollary 1: Compared to the centralized setting, the seller is less likely to add the GBW channel under the Agreement Price Contract, the Two-part Tariff Contract, and the Revenue-Sharing Contract.

When the seller and the GBW are decentralized firms, the GBW sets a group-buying price lower than the centralized setting (i.e., $p_{g}^{A^{*}}<p_{g}^{C^{*}}, p_{g}^{T^{*}}<p_{g}^{C^{*}}$, $\left.p_{g}^{R^{*}}<p_{g}^{C^{*}}\right)$. The GBW firm, in essence, competes with the seller by attracting the spot-market consumers to the GBW online channel. In addition, the GBW firm makes less sales effort than the centralized firm (i.e., $\gamma^{A^{*}}<\gamma^{C^{*}}, \gamma^{T^{*}}<\gamma^{C^{*}}, \gamma^{R^{*}}<\gamma^{C^{*}}$ ). Therefore, unless the GBW can bring enough new potential buyers and boost the seller's total sales, the seller runs the $\mathrm{O}$ model.

Proposition 6: Both the two-part tariff and the revenue-sharing contract cannot coordinate the profit of the seller and $G B W$.

When the whole profit of the seller and the GBW under two-part tariff contract or revenue-sharing contract is less than that under the centralized setting, both contracts cannot coordinate the profit of the seller and GBW.

\subsection{The pricing coordination under $O+G B W+R C$}

In this part, an attempt is made to coordinate the whole profit with the revenue-cost sharing contract (denoted by RC). The decision sequence is shown in Figure 5. With the RC contract, the seller and the GBW share their revenue with the proportion of $l$ and $(1-l)$, respectively; besides, the seller compensates the GBW $e^{\prime}\left(\gamma^{2} / 2\right)$ for the sales effort cost incurred at effort 


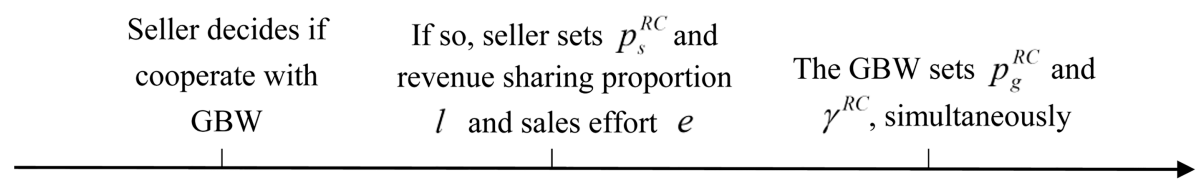

Figure 5. The sequence of events of the seller and Group-Buying Website (GBW) under the $\mathrm{O}+\mathrm{GBW}+\mathrm{RC}$ model.

level $\gamma$. Without loss of generality, let $e=e^{\prime} / 2$. Thus, the seller chooses parameter pair $(l, e)$. The sequence of events is as follows.

Under the revenue-cost sharing contract, the sales quantities through the online GBW channel and the traditional offline channel are respectively as follows:

$$
q_{g}^{R C}= \begin{cases}1-\frac{p_{g}^{R C}}{p_{o}}+b+\gamma^{R C}-p_{g}^{R C}, & p_{g}^{R C}<p_{o} \\ 0, & p_{g}^{R C} \geq p_{o}\end{cases}
$$

and:

$$
q_{s}^{R C}= \begin{cases}\frac{p_{g}^{R C}}{p_{o}}-p_{o}, & p_{g}^{R C}<p_{o} \\ 0, & p_{g}^{R C} \geq p_{o}\end{cases}
$$

The firms' maximization problems in the seller-leader case under the revenue-cost sharing contract can be formulated as follows:

$$
\begin{aligned}
& \left\{\begin{aligned}
\max _{p_{s}^{R C}, l, e} \Pi_{s}^{R C}= & \left(l \cdot p_{g}^{R C}+p_{s}^{R C}\right) q_{g}^{R C}+p_{o} \cdot q_{s}^{R C} \\
& -e \cdot\left(\gamma^{R C}\right)^{2} \\
\max _{p_{g}^{R C}, \gamma}^{R C} \Pi_{g}^{R C}= & {\left[(1-l) p_{g}^{R C}-p_{s}^{R C}\right] q_{g}^{R C} } \\
& -\frac{\left(\gamma^{R C}\right)^{2}}{2}+e \cdot\left(\gamma^{R C}\right)^{2}
\end{aligned}\right. \\
& \text { s.t.: } \quad q_{g}^{R C} \geq 0, \\
& \quad q_{s}^{R C} \geq 0 .
\end{aligned}
$$

All the results are summarized in Lemma 5.

Lemma 5: When taking the revenue-cost sharing contract:

1. The seller's optimal agreement price, sales compensation, and revenue-sharing proportion are $p_{s}^{R C^{*}}=$ $0, e^{*}=\frac{1}{2}$, and $l^{*}=1$, respectively. The $G B W^{\prime} s$ optimal group-buying price and effort are $p_{g}^{R C^{*}}=$ $\frac{2+b}{5}$ and $\gamma^{R C^{*}}=\frac{2+b}{5}$;

2. The seller's optimal profit is $\Pi_{s}^{R C^{*}}=\frac{3+8 b+2 b^{2}}{20}$; the $G B W$ 's optimal profit is $\Pi_{g}^{R C^{*}}=0$; the whole profit $\Pi^{R C^{*}}=\frac{3+8 b+2 b^{2}}{20}$ of the seller and $G B W$ is.

The seller's sales quantity through the GBW channel and the traditional offline channel are $q_{g}^{R C^{*}}=$ $\frac{1+3 b}{5}$ and $q_{s}^{R C^{*}}=\frac{3+4 b}{10}$, respectively. The discount on GBW is $\beta^{R C^{*}}=\frac{P_{g}^{R C^{*}}}{p_{o}^{*}}=\frac{2(2+b)}{5}$.
Note that the profit of the seller and GBW can be coordinated under the revenue-cost sharing contract. Thus, the condition for the seller to sell through groupbuying channel under the revenue-cost sharing contract is the same as that in benchmark setting.

Proposition 7: The seller adopts the $O+G B W+R C$ model if $b \geq \frac{\sqrt{10}-2}{6}$ and adopts the $O$ model otherwise.

Thus, the profit of the seller and GBW can be coordinated under the revenue-cost sharing contract. Then, comparing optimal results of the seller and GBW under these three coordination contracts, we get Proposition 8.

Proposition 8: Under the $O+G B W+R$ model, the seller obtains the least profit and the whole profit is also the least. Under the $O+G B W+A$ model, the $G B W$ obtains higher profit than under other contracts. Under the $O+G B W+R C$ model, the seller and the $G B W$ obtain the largest total profit.

All the results are shown in Table 1. By analyzing the results under different scenarios, the following corollaries are obtained.

Corollary 2: As the website scale $b$ increases, the $G B W$ will make more efforts to attract new consumers.

Corollary 3: The seller will choose the group-buying strategy when the website scale $b$ is sufficiently large. As $b$ increases, both the seller and the $G B W$ will obtain more profits.

When the seller uses group buying, the profits of the seller and the GBW under all settings increase with the scale of the website, $b$. This explains the reality that the sellers prefer to cooperate with larger GBWs.

\section{Model extensions}

This section investigates how the size of experienced consumers $(a)$ and the unit cost $(c)$ influence the seller and website's decisions.

In the centralized setting, the group buying and offline demands are:

$$
q_{g}^{C}= \begin{cases}\left(1-\frac{p_{g}^{C}}{p_{o}}\right) a+b+\gamma^{C}-p_{g}^{C}, & p_{g}^{C}<p_{o} \\ 0, & p_{g}^{C} \geq p_{o}\end{cases}
$$


Table 1. The seller and GBW's optimal decisions under different scenarios.

\begin{tabular}{|c|c|c|c|c|c|}
\hline & $\begin{array}{l}\text { Centralized } \\
\text { setting }\end{array}$ & $\begin{array}{l}\text { Agreement } \\
\text { price } \\
\text { contract }\end{array}$ & $\begin{array}{l}\text { Two-part } \\
\text { tariff } \\
\text { contract }\end{array}$ & $\begin{array}{c}\text { Revenue-sharing } \\
\text { contract }\end{array}$ & $\begin{array}{c}\text { Revenue-cost } \\
\text { sharing contract }\end{array}$ \\
\hline Condition & $b \geq \frac{\sqrt{10}-2}{6}$ & $b \geq \frac{-11+5 \sqrt{6}}{3}$ & $b \geq \frac{10 \sqrt{7}-23}{9}$ & $b \geq \sqrt{6}-2$ & \\
\hline$p_{g}^{*}$ & $\frac{2+b}{5}$ & $\frac{12 b+14}{45}$ & $\frac{12 b+14}{45}$ & $\frac{2+b}{6}$ & \\
\hline$p_{s}{ }^{*}$ & - & $\frac{3 b+5}{18}$ & $\frac{3 b+5}{18}$ & 0 & 0 \\
\hline$\gamma^{*}$ & $\frac{2+b}{5}$ & $\frac{3 b+1}{30}$ & $\frac{3 b+1}{30}$ & 0 & $\frac{2+b}{5}$ \\
\hline$\beta^{*}$ & $\frac{2(2+b)}{5}$ & $\frac{28+24 b}{45}$ & $\frac{28+24 b}{45}$ & $\frac{2+b}{3}$ & $\frac{2(2+b)}{5}$ \\
\hline$q_{g}^{*}$ & $\frac{1+3 b}{5}$ & $\frac{3 b+1}{10}$ & $\frac{3 b+1}{10}$ & $\frac{1}{2}$ & $\frac{1+3 b}{5}$ \\
\hline$q_{s}^{*}$ & $\frac{3+4 b}{10}$ & $\frac{48 b+11}{90}$ & $\frac{48 b+11}{90}$ & $\frac{1+2 b}{6}$ & $\frac{3+4 b}{10}$ \\
\hline$\Pi_{s}^{*}$ & - & $\frac{9 b^{2}+66 b+16}{180}$ & $\frac{11+46 b+9 b^{2}}{120}$ & $\frac{b^{2}+4 b+1}{12}$ & $\frac{3+8 b+2 b^{2}}{20}$ \\
\hline$\Pi_{g}^{*}$ & - & $\frac{(3 b+1)^{2}}{360}$ & 0 & 0 & 0 \\
\hline$\Pi^{*}$ & $\frac{3+8 b+2 b^{2}}{20}$ & $\frac{11+46 b+9 b^{2}}{120}$ & $\frac{11+46 b+9 b^{2}}{120}$ & $\frac{b^{2}+4 b+1}{12}$ & $\frac{3+8 b+2 b^{2}}{20}$ \\
\hline
\end{tabular}

and:

$$
q_{s}^{C}= \begin{cases}\frac{p_{g}^{C}}{p_{o}} a-p_{o}, & p_{g}^{C}<p_{o} \\ 0, & p_{g}^{C} \geq p_{o}\end{cases}
$$

respectively. Then, the problem can be reformulated as follows:

$$
\begin{aligned}
\max _{p_{g}^{C}, \gamma} \Pi_{s}^{C} & =\left(p_{g}^{C}-c\right) \cdot q_{g}^{C}+\left(p_{o}-c\right) \cdot q_{s}^{C}-\frac{\gamma^{C 2}}{2}, \\
\text { s.t.: } \quad q_{g}^{C} & \geq 0 \\
q_{s}^{C} & \geq 0 .
\end{aligned}
$$

Under the Agreement Price Contract, the group-buying and offline demands are:

$$
q_{g}^{A}= \begin{cases}\left(1-\frac{p_{g}^{A}}{p_{o}}\right) a+b+\gamma^{A}-p_{g}^{A}, & p_{g}^{A}<p_{o} \\ 0, & p_{g}^{A} \geq p_{o}\end{cases}
$$

and:

$$
q_{s}^{A}= \begin{cases}\frac{p_{g}^{A}}{p_{o}} \cdot a-p_{o}, & p_{g}^{A}<p_{o} \\ 0, & p_{g}^{A} \geq p_{o}\end{cases}
$$

respectively. The profit of the seller and GBW can be reformulated as follows:

$$
\begin{aligned}
& \left\{\begin{array}{l}
\max _{p_{s}^{A}} \Pi_{s}^{A}=\left(p_{s}^{A}-c\right) \cdot q_{g}^{A}+\left(p_{o}-c\right) \cdot q_{s}^{A} \\
\max _{p_{g}^{A}, \gamma^{A}} \Pi_{g}^{A}=\left(p_{g}^{A}-p_{s}^{A}\right) \cdot q_{g}^{A}-\frac{\left(\gamma^{A}\right)^{2}}{2}
\end{array}\right. \\
& \text { s.t.: } \quad q_{g}^{A} \geq 0, \\
& \qquad q_{s}^{A} \geq 0 .
\end{aligned}
$$

Under the $\mathrm{O}+\mathrm{GBW}+\mathrm{RC}$ model, the group buying and offline demands are:

$$
q_{g}^{R C}= \begin{cases}\left(1-\frac{p_{g}^{R C}}{p_{o}}\right) a+b+\gamma^{R C}-p_{g}^{R C}, & p_{g}^{R C}<p_{o} \\ 0, & p_{g}^{B R C} \geq p_{o}\end{cases}
$$


Table 2. The optimal results of the seller and Group-Buying Website (GBW) under different scenarios.

\begin{tabular}{|c|c|c|c|}
\hline & Centralized setting & Agreement price contract & Revenue-cost sharing contrac \\
\hline \multirow{3}{*}{ Conditions $b \geq$} & $3 a c-2 a^{2}+c^{2}$ & \multirow{3}{*}{$b \geq \frac{+(a-c)(5 a+c) \sqrt{2 a(3 a+c)}}{(3 a+c)(a+c)}$} & \multirow{2}{*}{$\begin{array}{l}3 a c-2 a^{2}+c^{2}+\left(5 a^{2}\right. \\
\left.-c^{2}-4 a c\right) \sqrt{a /(5 a+c)}\end{array}$} \\
\hline & $+\left(5 a^{2}-c^{2}-4 a c\right) \sqrt{\frac{a}{5 a+c}}$ & & \\
\hline & $a+c$ & & $a+c$ \\
\hline \multirow{3}{*}{$p_{g}^{*}$} & & $(a+c)\left(12 a^{2} b+2 a c^{2}+12 a^{2} c\right.$ & \\
\hline & $(a+c)(2 a+b)$ & $\left.+12 a c^{2}+b c^{2}+14 a^{3}+7 a b c\right)$ & $(a+c)(2 a+b)$ \\
\hline & $5 a+c$ & $(3 a+c)^{2}(5 a+c)$ & $5 a+c$ \\
\hline$n^{*}$ & & $(a+c)\left(3 a b+6 a c+b c+5 a^{2}+c^{2}\right)$ & \\
\hline$p_{s}$ & - & $2(3 a+c)^{2}$ & 0 \\
\hline \multirow{2}{*}{$\gamma^{*}$} & $2 a^{2}-3 a c+a b-c^{2}+b c$ & $(a+c)\left(3 a b-4 a c+b c+a^{2}-c^{2}\right)$ & $2 a^{2}-3 a c+a b-c^{2}+b c$ \\
\hline & $5 a+c$ & $2(3 a+c)(5 a+c)$ & $5 a+c$ \\
\hline \multirow{3}{*}{$\beta^{*}$} & & $28 a^{3}+24 a^{2} c+24 a^{2} b$ & \\
\hline & $2(2 a+b)$ & $+4 a c^{2}+14 a b c+2 b c^{2}$ & $2(2 a+b)$ \\
\hline & $5 a+c$ & $(3 a+c)^{2}(5 a+c)$ & $5 a+c$ \\
\hline \multirow[b]{2}{*}{$q_{g}^{*}$} & $a^{2}-4 a c+3 a b-c^{2}+b c$ & $b(3 a+c)-4 a c+a^{2}-c^{2}$ & $a^{2}-4 a c+3 a b-c^{2}+b c$ \\
\hline & $5 a+c$ & $2(5 a+c)$ & $5 a+c$ \\
\hline \multirow{3}{*}{$q_{s}^{*}$} & & $4 a b(4 a+c)(3 a+c)+\left(a^{2}-4 a c\right.$ & \\
\hline & $3 a^{2}-6 a c+4 a b-c^{2}$ & $\left.-c^{2}\right)\left(8 a c+11 a^{2}+c^{2}\right)$ & $3 a^{2}-6 a c+4 a b-c^{2}$ \\
\hline & $\frac{2(5 a+c)}{2}$ & $2(5 a+c)(3 a+c)^{2}$ & $2(5 a+c)$ \\
\hline \multirow{4}{*}{$\Pi_{s}^{*}$} & \multirow{4}{*}{ - } & $(a+c)(3 a+c)^{2} b^{2}+2 b(3 a+c)\left(-7 a c^{2}\right.$ & $3 a^{3}+8 a^{2} b-13 a^{2} c$ \\
\hline & & $\left.-11 a^{2} c+11 a^{3}-c^{3}\right)+2\left(a^{2}-4 a c\right.$ & $+2 a b^{2}-12 a b c+11 a c^{2}$ \\
\hline & & $\left.-c^{2}\right)\left(-6 a c^{2}-5 a^{2} c+8 a^{3}-c^{3}\right)$ & $+2 b^{2} c-4 b c^{2}+3 c^{3}$ \\
\hline & & $4(5 a+c)(3 a+c)^{2}$ & $4(5 a+c)$ \\
\hline$\Pi_{g}^{*}$ & - & $\frac{(a+c)\left(3 a b+b c-4 a c+a^{2}-c^{2}\right)^{2}}{8(5 a+c)(3 a+c)^{2}}$ & 0 \\
\hline
\end{tabular}

and:

$$
q_{s}^{R C}= \begin{cases}\frac{p_{g}^{R C}}{p_{o}} a-p_{o}, & p_{g}^{R C}<p_{o} \\ 0, & p_{g}^{R C} \geq p_{o}\end{cases}
$$

respectively. The profit of the seller and GBW can be reformulated as follows:

$$
\left\{\begin{aligned}
\max _{p_{s}^{R C}, l, e} \Pi_{s}^{R C}= & \left(l \cdot p_{g}^{R C}+p_{s}^{R C}-c\right) q_{g}^{R C} \\
+ & \left(p_{o}-c\right) \cdot q_{s}^{R C}-e \cdot\left(\gamma^{R C}\right)^{2} \\
\max _{p_{g}^{R C}, \gamma^{R C} \Pi_{g}^{R C}=} & {\left[(1-l) p_{g}^{R C}-p_{s}^{R C}\right] q_{g}^{R C} } \\
& -\frac{\left(\gamma^{R C}\right)^{2}}{2}+e \cdot\left(\gamma^{R C}\right)^{2}
\end{aligned}\right.
$$

$$
\begin{array}{ll}
\text { s.t.: } & q_{g}^{R C} \geq 0 \\
& q_{s}^{R C} \geq 0 .
\end{array}
$$

Solving the profit maximization problems of the GBW and the seller under different scenarios, we can get all the optimal decisions on group-buying prices, agreement prices, promotion effort, etc. All the optimal results are listed in Table 2 and more details are presented in Appendix B.

\subsection{The impact of the service/product cost for the seller and the $G B W$}

Former studies have shown that product or service unit cost plays a key role in the group-buying strategy. 
Edelman et al. (2016) [13] pointed out that when providing a service or product with lower marginal costs, the GBW will benefit more. The statistics of Tuan 800 (one of the largest GBWs in China) in June 2015 show that catering and entertainment (such as cinema tickets and amusement) are the top two group-buying categories, which account for $61.89 \%$ and $16.78 \%$ of the total sales, respectively. However, their average deal prices are less than 80 and $50 \mathrm{RMB}$, respectively. When talking about their development orientation, Wang Xing, the CEO of the largest GBW, emphasizes that "Meituan will insist on providing service group buying rather than product group buying, because the low marginal cost of service group buying will be the most attractive in price and quality" [28]. Therefore, this section further investigates the impact of unit cost on the optimal decisions and profits for the seller and the website.

Besides, for all the results shown in Table 2, the unit cost satisfies the condition that $0 \leq c \leq a$. Table 2 lists the optimal results of the seller and the website under different marketing scenarios. Based on these results, we will analyze the impact of product cost and experienced offline consumers for the optimal strategies of the seller and the website.

Proposition 9: As the unit cost $c$ increases, the following will hold:

1. The $G B W$ will set a higher group-buying price and the seller will set a higher agreement price;

2. $\beta$ decreases, which means the group-buying discount is larger;

3. Group-buying and offline demands both decrease;

4. The website's and the seller's profits decrease.

As the unit cost increases, the seller will set a higher offline regular price $\left(\frac{\partial p_{o}}{\partial c}>0\right)$ and charge the website a higher agreement price $\left(\frac{\partial p_{s}}{\partial c}>0\right)$. The website will choose a higher group-buying price $\left(\frac{\partial p_{g}}{\partial c}>0\right)$ accordingly; however, its increase rate is much smaller than the offline regular price $\left(\frac{\partial p_{o}}{\partial c}>\frac{\partial p_{g}}{\partial c}\right)$. Therefore, $\beta$ decreases, which means that the group-buying discount is larger. Although increased offline regular prices will transfer more experienced consumers to the GBW, the size of new consumers that the website attracts with the group-buying price decreases. Overall, the groupbuying demand decreases $\left(\frac{\partial q_{g}^{C}}{\partial c}<0, \frac{\partial q_{g}^{A}}{\partial c}<0\right)$. The offline demand will apparently decrease due to a higher transfer rate from the offline channel to the groupbuying channel $\left(\frac{\partial q_{s}^{C}}{\partial c}<0, \frac{\partial q_{s}^{A}}{\partial c}<0\right)$. The seller's and the website's profits suffer from the higher unit cost and the corresponding shrunken demands from both channels.
As the seller and the website both tend to offer products or services with lower marginal costs, it is more likely for a seller with a relatively low unit cost to choose the group-buying strategy. This explains the phenomenon that catering and entertainment groupbuying products account for an overwhelming majority of the group-buying market. In 2010, Tuan 800 investigated the average price per group-buying transaction of the top 10 Chinese GBW. The results show that groupbuying products with a price lower than $50 \mathrm{RMB}$ are more attractive.

\subsection{The impact of the experienced consumer size for the seller and the $G B W$}

The GBW prefers to consociate with sellers with a larger number of experienced consumers, better consumer evaluation, and longer operating history in the local area since these sellers generally invest more in their brand maintenance and product or service promotion. They will not damage their brand for petty profits [29]. This section investigates how the size of experienced consumers affects the group-buying market and the decisions of both sellers and websites. Based on the optimal results shown in Table 2, the following results can be obtained.

Proposition 10: As the size of experienced consumers a increases:

1. The seller will set a higher agreement price and the $G B W$ will set a higher group-buying price;

2. $\beta$ decreases, which means the group-buying discount is deeper;

3. The $G B W$ will enhance the promotion effort $\gamma$;

4. The group-buying demand and the offline demand increase;

5. Both the seller and the website will obtain higher profit.

With the larger number of experienced consumers, the seller will set a higher offline regular price $\left(\frac{\partial p_{o}}{\partial a}>0\right)$. Other than that, the seller will charge the website a higher agreement price $\left(\frac{\partial q_{s}^{C}}{\partial a}>0\right)$, which induces the website to enhance group-buying price $\left(\frac{\partial p_{g}^{A}}{\partial a}>0, \frac{\partial p_{g}^{C}}{\partial a}>0, \frac{\partial p_{g}^{B}}{\partial a}>0\right)$. The increase rate of offline regular price is higher than that of group-buying price $\left(\frac{\partial p_{o}}{\partial a}>\frac{\partial p_{g}^{A, B, C}}{\partial a}\right)$; thus, $\beta$ decreases $\left(\frac{\partial \beta^{C}}{\partial a}<0, \frac{\partial \beta^{A}}{\partial a}<0, \frac{\partial \beta^{B}}{\partial a}<0\right)$, which means that the group-buying discount is deeper. When taking $\mathrm{O}+\mathrm{GBW}$ model, more experienced consumers move on to the GBW channel and the website will attract more new consumers; thus, group-buying demand increases $\left(\frac{\partial q_{g}^{A}}{\partial a}>0\right)$. Since both the seller and the website's profits increase with increasing the number of experienced 
consumers, the website will tend to work with a seller with a larger number of experienced consumers.

\section{Numerical study}

The primary objectives of this numerical study are three-fold. First, we would like to verify the results that have been proven in the previous section. It was found that the seller would choose the group-buying strategy when the website scale $b$ was sufficiently large. Moreover, as $b$ increases, both the seller and the GBW will obtain more profits, thus verifying and complementing Propositions 1, 2, 3, 5, and 7. Second, the advantage and profitability of different contracts, the $O+G B W$ model, $O+G B W+T$ model, $O+G B W+R$ model, and $O+G B W+R C$ model, should be compared with the centralized setting. Third, it is desired to verify the impact of group-buying service/product cost and experienced consumer number from different perspectives such as the agreement price, group-buying price, group-buying discount $\beta$, group-buying effort $\gamma$, and the profit of seller and GBW.

\subsection{The impact of $G B W$ scale $b$}

Let's first start by presenting the results of the profit of the seller and website with $b$ changing under different contracts. As shown in Figure 6 , when $b$ is larger than a threshold value, the seller will obtain higher profit than operating only offline channel. This further verifies Propositions 1, 2, 3, 5, and 7. Moreover, it was found that both the seller and the website would obtain higher profit with increasing $b$. Thus, we also further verify Corollary 3.

\subsection{The profitability comparison between contracts}

In this subsection, to further understand the profitability of different contracts for both the seller and the website, we desire to present the seller and the website's profits with different website scales $b$.

As shown in Figure 7(a), the seller can always get higher profit under the centralized setting than that under agreement price contract, two-part tariff contract, and revenue-sharing contract, no matter how much $b$ changes. This further verifies Corollary 1. In addition, comparing seller's profit under agreement price contract, two-part tariff contract, revenue-sharing contract, and revenue-cost sharing contract, we can find that the seller obtains the least profit under the $\mathrm{O}+\mathrm{GBW}+\mathrm{R}$ model.

Figure 7(b) obviously shows that, under the $\mathrm{O}+\mathrm{GBW}+\mathrm{A}$ model, the GBW obtains higher profit than that under other contracts. In Figure $7(\mathrm{c})$, comparing the total profit of the seller and website under $\mathrm{O}+\mathrm{GBW}+\mathrm{T}$ and $\mathrm{O}+\mathrm{GBW}+\mathrm{R}$, we prove that both the two-part tariff and the revenue-sharing contract cannot coordinate the profit of the seller and GBW, further verifying Proposition 6. Besides, under the $\mathrm{O}+\mathrm{GBW}+\mathrm{A}$ model, the GBW obtains higher profit than that under other contracts; in addition, under the $\mathrm{O}+\mathrm{GBW}+\mathrm{RC}$ model, the seller

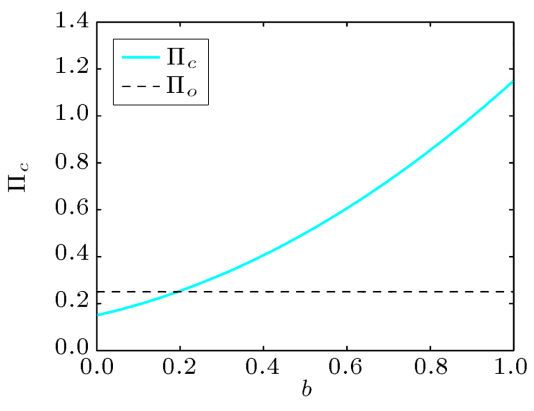

(a)

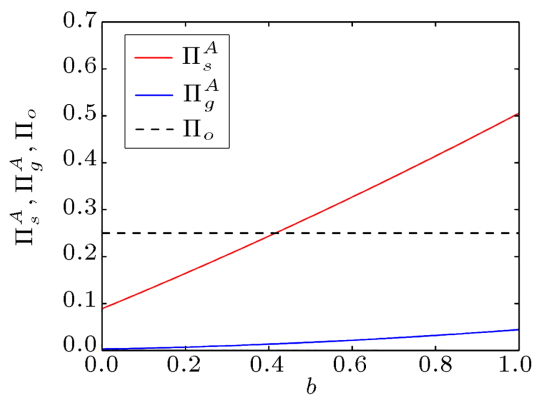

(b)

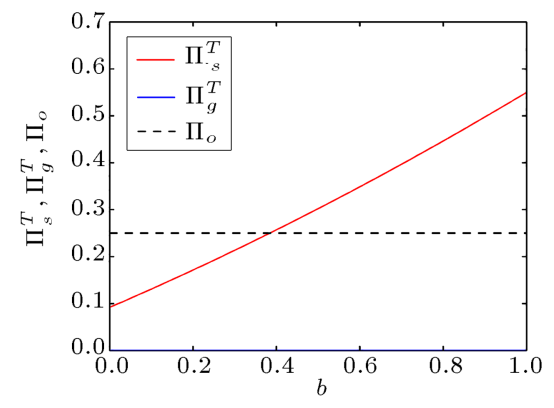

(c)

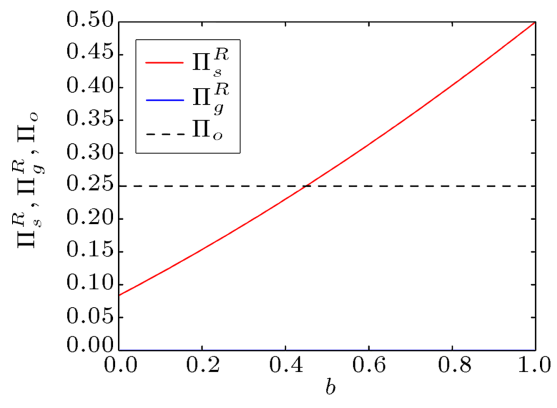

(d)

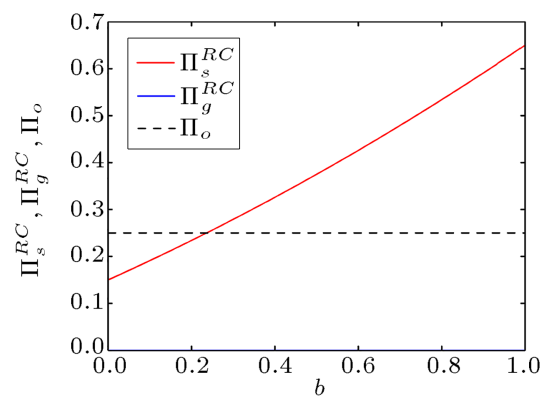

(e)

Figure 6. The impact of $b$ on the seller and website's profit. 


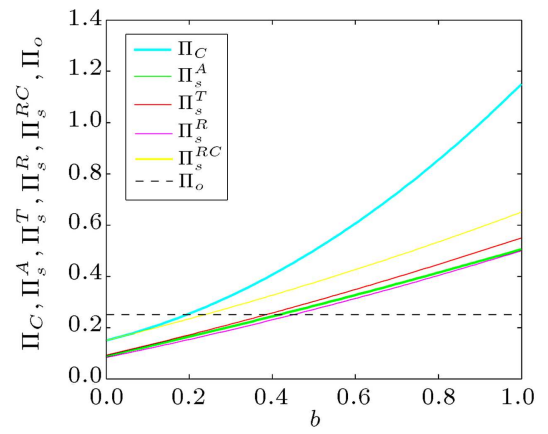

(a)

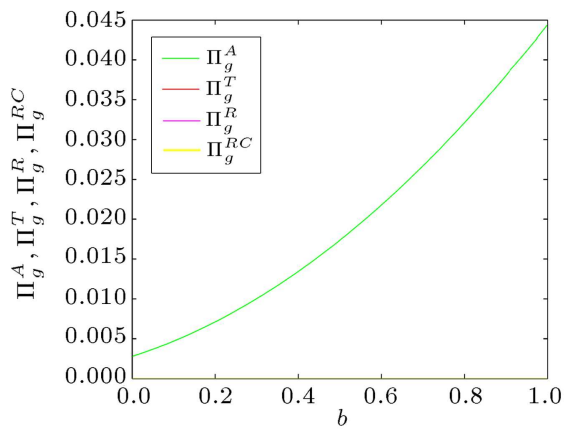

(b)

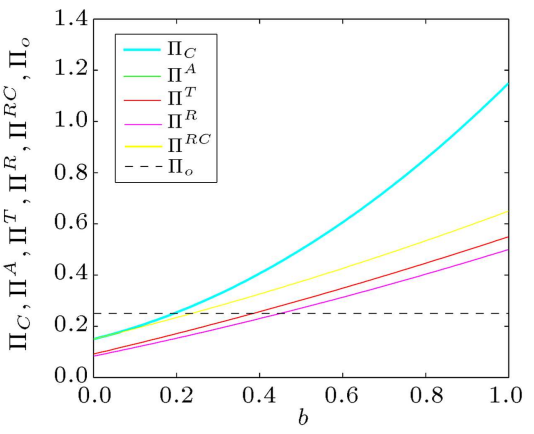

(c)

Figure 7. The profitability comparison under different contracts.

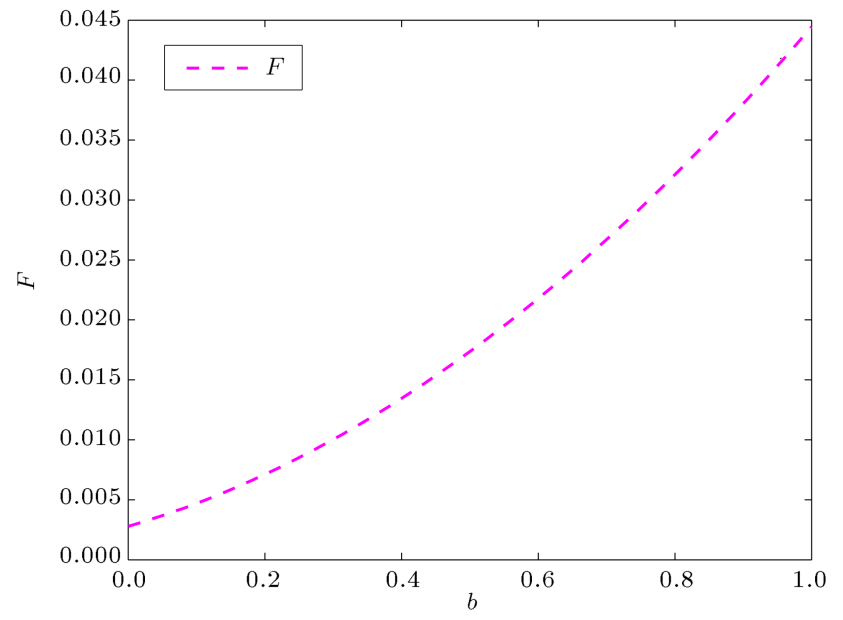

Figure 8. The impact on $F$ with $b$ increasing.

and the GBW obtain the largest total profit. Thus, Proposition 8 is also verified.

\subsection{The impact of GB service/product cost and experienced consumer size}

In this part, more numerical studies are conducted to further reveal how the major parameters affect the performance of seller and website under different contracts.

In Figure 8, with $b$ increasing, the seller will set higher fixed payment, which is exactly shown in Proposition 4. Figure 9 shows clearly that the GBW will offer more effort with its scale increasing. Thus, this exactly explains Corollary 2.

To analyze the impact of GB service/product cost $c$ and experienced consumer size $a$, we assume that $a=1$ and $b=1$ when focusing on the impact of $c$ and, also, assume that $c=0$ and $b=1$ when investigating the impact of $a$.

In Figure 10(a), the GB price and agreement price are always increasing with $c$. In Figure 10(b), $\beta$ is decreasing with $c$, which means that the GB discount is getting larger. Figure $10(\mathrm{c}),(\mathrm{d})$, and (e) obviously show that the GB demand, offline demand, and the

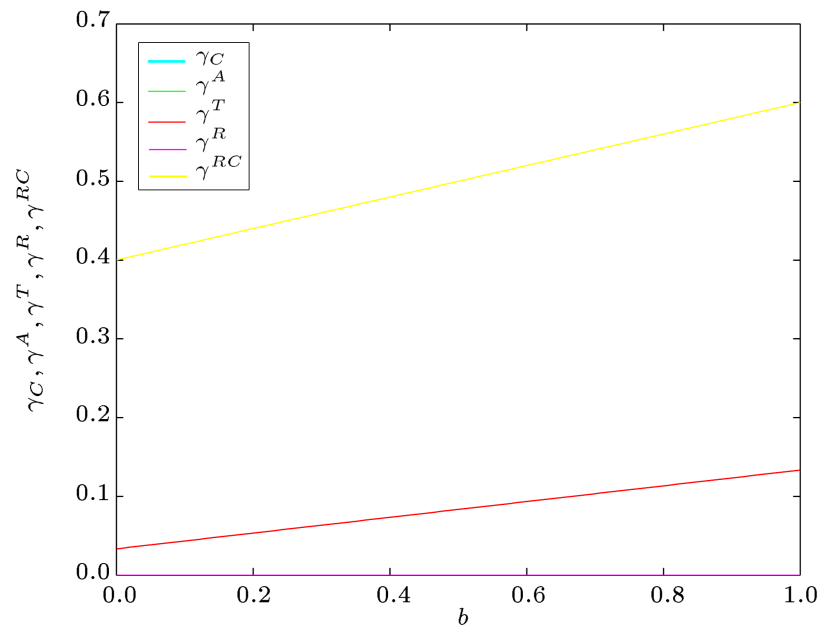

Figure 9. The impact on $\gamma$ with $b$ increasing.

profit of the seller and GBW are always decreasing. Thus, Proposition 9 was verified in Section 6 .

\section{Conclusion}

This paper considered a seller who can choose to sell through a GBW, in addition to its traditional offline channel. GBW can make costly efforts to run group buying, which will increase the number of new consumers. The seller's decision on whether or not to implement group buying and how the contract between the seller and GBW impacts the seller's profitability was studied.

It was found that when the GBW had an attractive consumer base size, the seller would choose to use group buying. When the seller and the GBW took twopart tariff or revenue-sharing contracts, the total profit of the two channels was less than that of a centralized firm, that is, the two contracts could not coordinate the system. Based on this finding, a revenue-cost sharing contract that can coordinate the two channels was also proposed.

This study adopted the analytical modeling and 


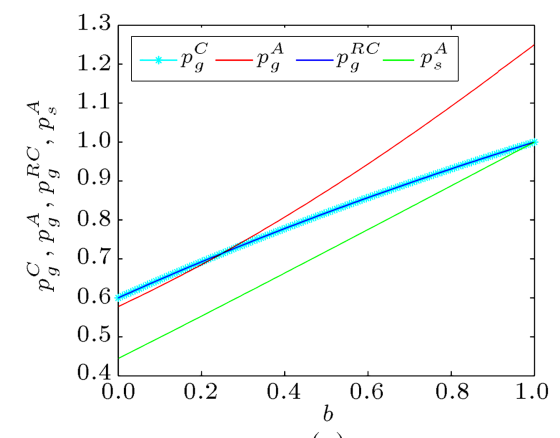

(a)

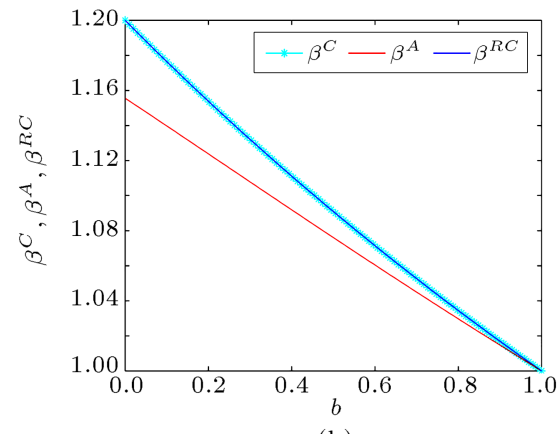

(b)

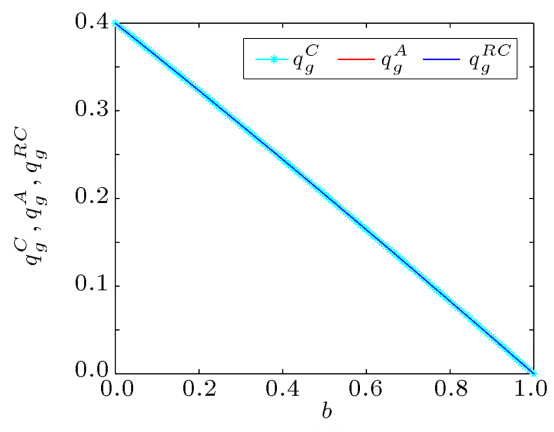

(c)

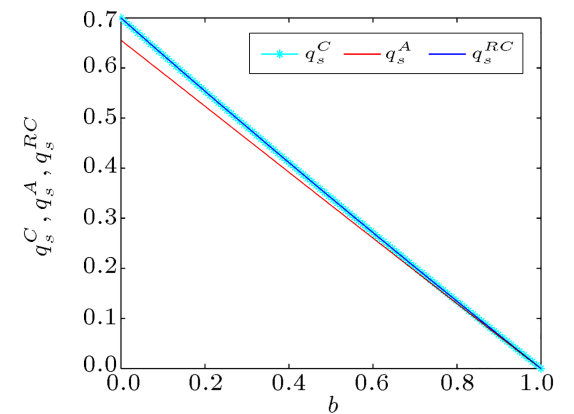

(d)

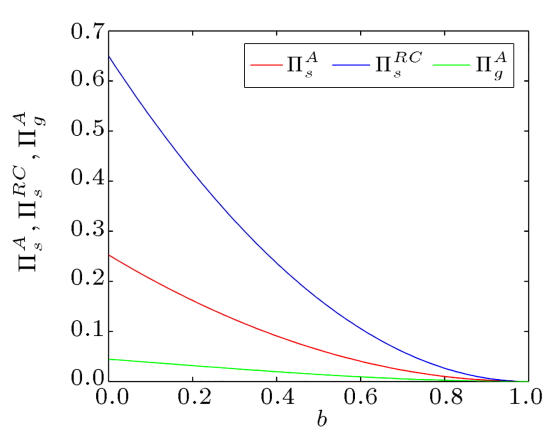

(e)

Figure 10. The impact of Group-Buying (GB) service/product cost $c(a=1$ and $b=1)$.

the game theoretic approach. Although such an analysis enables us to generate managerial insights, it usually lacks data corroboration. In the future research, one can collect corresponding data to examine the exact relation between the group-buying promotions and a seller's profitability. Besides, in the future, it is worth exploring some other easily implementable contracts that may better coordinate the revenue of the seller and the website.

\section{Acknowledgement}

This work was supported in part by the National Natural Science Foundation of China (71471062, 71431004, 71671067, and 71301050), the Shanghai Pujiang Program (17PJC023, 13PJC022), and the Fundamental Research Funds for the Central Universities.

\section{References}

1. Dholakia, U.M. "How businesses fare with daily deals as they gain experience: A multi-time period study of daily deal performance", Working Paper., Rice University, Houston, USA (2012).

2. Dholakia, U.M. "What makes groupon promotions profitable for businesses?", Working Paper, Rice University, Houston, USA (2011a).

3. Sahin, H. and Topal, B. "Impact of information technology on business performance: Integrated structural equation modelling and artificial neural network approach", Sci. Iran., 25(3), pp. 1272-1280 (2018).
4. Shugan, S.M. "The impact of advancing technology on marketing and academic research", Marketing Sci., 23(4), pp. 469-475 (2004).

5. Wang, J.J., Zhao, X., and Li, J.J. "Group buying: A strategic form of consumer collective", J. Retailing., 89(3), pp. 338-351 (2013).

6. Chen, J., Chen, X., and Song, X. "Bidder's strategy under group-buying auction on the internet", IEEE. T. Syst. Man. CY-S., 32(6), pp. 680-690 (2002).

7. Anand, K.S. and Aron, R. "Group buying on the web: A comparison of price-discovery mechanisms", Management Sci., 49(11), pp. 1546-1562 (2003).

8. Chen, J., Chen, X., and Song, X. "Comparison of the group-buying auction and the fixed pricing mechanism", Decision Support Syst., 43(2), pp. 445-459 (2007).

9. Chen, J., Chen, X., Kauffman, R.J., and Song, X. "Should we collude? Analyzing the benefits of bidder cooperation in online group-buying auctions", Electron. Commer. R. A., 8(4), pp. 191-202 (2009).

10. Dholakia, U.M. and Tsabar, G.A. "Startup's experience with running a groupon promotion", Working Paper, Rice University, Houston, USA (2011b).

11. Heo, C.Y. "Exploring group-buying platforms for restaurant revenue management", Int. J. Hosp. Manag, 52, pp. 154-159 (2016).

12. $\mathrm{Wu}, \mathrm{Y}$. and $\mathrm{Zhu}, \mathrm{L}$. "Joint quality and pricing decisions for service online group-buying strategy", Electron. Commer. R. A., 25, pp. 1-15 (2017).

13. Edelman, B., Jaffe, S., and Kominers, S.D. "To groupon or not to groupon: the profitability of deep discounts", Market. Lett., 27(1), pp. 39-53 (2016). 
14. Gao, F. and Chen, J. "The role of discount vouchers in market with customer valuation uncertainty", Prod. Oper. Manag., 24(4), pp. 665-679 (2015).

15. Ni, G., Luo, L., Xu, Y., Xu, J., and Dong, Y. "Optimal decisions on group buying option with a posted retail price and heterogeneous demand", Electron. Commer. R. A., 14(1), pp. 23-33 (2015).

16. Taleizadeh, A.A., Satarian, F., and Jamili, A. "Optimal multi-discount selling prices schedule for deteriorating product", Sci. Iran., 22(6), pp. 2595-2603 (2015).

17. Zhao, M., Wang, Y., and Gan, X. "Signalling effect of daily deal promotion for a start-up service provider", J. Oper. Res. Soc., 67(2), pp. 280-293 (2016).

18. Kauffman, R.J. and Wang, B. "Bid together, buy together: on the efficacy of group-buying business models in Internet-based selling", In Handbook of Electronic Commerce in Business and Society, P.B. Lowry, J.O. Cherrington, and R.R. Watson, Eds., CRC Press, Boca Raton, FL. pp. 99-137 (2002).

19. Zhao, N. "Every day there averagely are 5.9 group-buying websites going out of business in China", Retrieved January 6, Translated from Chinese language, http://www.21cbh.com/HTML/201210-25/0NNDE5XzU0NzM0Ng.html. (2013).

20. $\mathrm{Pu}, \mathrm{X}$., Gong, L., and Han, X. "Consumer free riding: Coordinating sales effort in a dual-channel supply chain", Electron. Commer. R. A., 22, pp. 1-12 (2017).

21. Tran, T. and Desiraju, R. "Group-buying and channel coordination under asymmetric information", Eur. J. Oper. Res., 256(1), pp. 68-75 (2017).

22. Ni, G., Xu, Y., Xu, J., and Dong, Y. "Optimal decisions for fixed-price group-buying business originated in China: a game theoretic perspective", Int. J. Prod. Res., 53(10), pp. 2995-3005 (2015).

23. Bhardwaj, P. and Sajeesh, S. "Strategic revenue sharing with daily deal sites: A competitive analysis", Decision Sci., 48(6), pp. 1228-1261 (2017).

24. Subramanian, U. and Rao, R.C. "Leveraging experienced consumers to attract new consumers: An equilibrium analysis of displaying deal sales by daily deal websites", Management Sci., 62(12), pp. 35553575 (2016).

25. Cao, J., So, K.C., and Yin, S. "Impact of an 'onlineto-store' channel on demand allocation, pricing and profitability", Eur. J. Oper. Res., 248(1), pp. 234-245 (2016).

26. Chen, J., Liang, L., Yao, D., and Sun, S. "Price and quality decisions in dual-channel supply chains", Eur. J. Oper. Res., 259(3), pp. 935-948 (2016).

27. Zhang, P., He, Y., and Shi, C. "Retailer's channel structure choice: Online channel, offline channel, or dual channels?", Int. J. Prod. Econ., 191, pp. 37-50 (2017).
28. Wang, X. "Insisting on providing group-buying services but not group-buying products", http://zixun. tuan800.com/a/jingyingshidian/20120407/30168. html (2012).

29. Tuan 800. "The Chinese group-buying market statics report in June 2015", http://zixun. tuan800.com/a/ tuangoushujubaogao/20150727/50585. html (2015).

\section{Appendix A}

\section{Centralized setting}

Proof of Lemma 1: Taking the first-order and second derivative of $\Pi^{C}$ with respect to $p_{g}^{C}$ and $\gamma^{C}$ respectively, we can get:

$$
\begin{aligned}
& \frac{\partial \Pi^{C}}{\partial p_{g}^{C}}=\frac{\left(2+b+\gamma^{C}\right) p_{o}-2\left(1+p_{o}\right) p_{g}^{C}}{p_{o}} \\
& \frac{\partial \Pi^{C}}{\partial \gamma^{C}}=p_{g}^{C}-\gamma^{C}, \quad \frac{\partial^{2} \Pi^{C}}{\partial p_{g}^{C^{2}}}=-2\left(\frac{1}{p_{o}}+1\right), \\
& \frac{\partial^{2} \Pi^{C}}{\partial \gamma^{C^{2}}}=-1, \quad \frac{\partial^{2} \Pi^{C}}{\partial p_{g}^{C} \partial \gamma^{C}}=1, \\
& \frac{\partial^{2} \Pi^{C}}{\partial \gamma^{C} \partial p_{g}^{C}}=1 .
\end{aligned}
$$

Then, we can get the Hesse Matrix of $\Pi^{C}$ about $p_{g}^{C}$ and $\gamma^{C}, H\left(\Pi^{C}\right)=\left[\begin{array}{cc}-2\left(\frac{a}{p_{o}}+1\right) & 1 \\ 1 & -1\end{array}\right]$. This Hesse Matrix is a negative definite matrix, which means $\Pi^{C}$ has a maximum value about $p_{g}^{C}$ and $\gamma^{C}$.

When the seller and website corporate to provide group-buying products, the online and offline demand, respectively, are:

$$
\begin{aligned}
& q_{g}^{C}= \begin{cases}1-\frac{p_{g}^{C}}{p_{o}}+b+\gamma^{C}-p_{g}^{C}, & p_{g}^{C}<p_{o} \\
0, & p_{g}^{C} \geq p_{o}\end{cases} \\
& q_{s}^{C}=\left\{\begin{array}{ll}
\frac{p_{g}^{C}}{p_{o}}-p_{o}, & p_{g}^{C}<p_{o} \\
0, & p_{g}^{C} \geq p_{o}
\end{array} .\right.
\end{aligned}
$$

Setting $q_{g}^{C}>0$ and $q_{s}^{C}>0$ yields $p_{o}^{2}<p_{g}^{C}<\frac{p_{o}(b+1)}{1+p_{o}}$, and $\frac{1}{4}<p_{g}^{C}<\frac{b+1}{3}$. Maximizing Eq. (1) with respect to $p_{g}^{C}$ and $\gamma^{C}$ yields $p_{g}^{C^{*}}=\frac{2+b}{5}$ and $\gamma^{C^{*}}=\frac{2+b}{5}$. Then, the optimal group-buying discount should be $\beta^{C^{*}}=\frac{p_{g}^{C}}{p_{o}}=$ $\frac{2(2+b)}{5}$ and the online and offline demand, respectively, are $q_{g}^{C^{*}}=\frac{1+3 b}{5}$ and $q_{s}^{C^{*}}=\frac{3+4 b}{10}$. The optimal profit under centralized setting should be $\Pi^{C^{*}}=\frac{3+8 b+12 b^{2}}{20}$. For all the results, they all satisfy the condition $b \geq$ $\frac{\sqrt{10}-2}{6}$.

Proof of Proposition 1: Only when the seller obtains higher profit under the $\mathrm{O}+\mathrm{GBW}$ model than 
under the offline channel, he chooses $\mathrm{O}+\mathrm{GBW}$ which means $\Pi^{C^{*}}=\frac{3+8 b+12 b^{2}}{20}>\Pi_{o}^{*}=\frac{1}{4}$, then $b \geq \frac{\sqrt{10}-2}{6}$ is obtained.

\section{Decentralized setting-agreement price contract}

Proof of Lemma 2: When operating group-buying, the online and offline demand are given by:

$$
q_{g}^{A}= \begin{cases}1-\frac{p_{g}^{A A}}{p_{o}}+b+\gamma^{A}-p_{g}^{A}, & p_{g}^{A}<p_{o} \\ 0, & p_{g}^{A} \geq p_{o}\end{cases}
$$

and:

$$
q_{s}^{A}=\left\{\begin{array}{ll}
\frac{p_{g}^{A}}{p_{o}}-p_{o}, & p_{g}^{A}<p_{o} \\
0, & p_{g}^{A} \geq p_{o}
\end{array} .\right.
$$

Setting $q_{g}^{A}>0$ and $q_{s}^{A}>0$, we get $p_{o}^{2}<p_{g}^{A}<$ $\frac{(1+b+\gamma) p_{o}}{1+p_{o}} \leq \frac{(1+b) p_{o}}{1+p_{o}}$. Substituting $p_{o}$ into the results, we get $\frac{1}{4}<p_{g}^{A}<\frac{b+1}{3}$. Under this condition, the profit of the seller and website are given by:

$$
\Pi_{g}^{A}=\left(p_{g}^{A}-p_{s}^{A}\right)\left(1-\frac{p_{g}^{A}}{p_{o}}+b+\gamma^{A}-p_{g}^{A}\right)-\frac{\left(\gamma^{A}\right)^{2}}{2},
$$

and:

$$
\Pi_{s}^{A}=p_{s}^{A} \cdot\left(1-\frac{p_{g}^{A}}{p_{o}}+b+\gamma^{A}-p_{g}^{A}\right)+p_{o} \cdot \frac{p_{g}^{A}}{p_{o}}-p_{o} .
$$

Using the backward induction technique, we firstly solve maximization problem of $\Pi_{g}^{A}$ with respect to $p_{g}^{A}$ and $\gamma^{A}$. Then substituting the optimal results of $p_{g}^{A}$ and $\gamma^{A}$ into $\Pi_{s}^{A}$, we can solve the optimal result of agreement price $p_{s}^{A}$, and then the optimal profit of the seller.

Taking the first-order and second derivative of $\Pi_{g}^{A}$ with respect to $p_{g}^{A}$ and $\gamma^{A}$, we can get:

$$
\begin{aligned}
& \frac{\partial \Pi_{g}^{A}}{\partial p_{g}^{s}}=1+b+\gamma^{A}+\left(\frac{1}{p_{o}}+1\right) p_{s}^{A}-2\left(\frac{1}{p_{o}}+1\right) p_{g}^{A}, \\
& \frac{\partial \Pi_{g}^{A}}{\partial \gamma^{s}}=p_{g}^{A}-p_{s}^{A}-\gamma^{A}, \quad \frac{\partial^{2} \Pi_{g}^{A}}{\partial p_{g}^{A} \partial \gamma^{A}}=1, \\
& \frac{\partial^{2} \Pi_{g}^{A}}{\partial \gamma^{A} \partial p_{g}^{A}}=1, \quad \frac{\partial^{2} \Pi_{g}^{A}}{\partial p_{g}^{A^{2}}}=-2\left(\frac{1}{p_{o}}+1\right), \\
& \frac{\partial^{2} \Pi_{g}^{A}}{\partial \gamma^{A^{2}}}=-1 .
\end{aligned}
$$

The Hesse matrix of $\Pi_{g}^{A}$ about $p_{g}^{A}$ and $\gamma^{A}$ is:

$$
H\left(\Pi_{g}^{A}\right)=\left[\begin{array}{cc}
-2\left(\frac{1}{p_{o}}+1\right) & 1 \\
1 & -1
\end{array}\right],
$$

which is a negative definite matrix and means that there is a maximum value with respect to $\Pi_{g}^{A}$. Max- imizing Eq. (4) yields $p_{g}^{A *}\left(p_{s}^{A}\right)=\frac{(1+b) p_{o}+p_{s}^{A}}{2+p_{o}}$ and $\gamma^{A *}\left(p_{s}^{A}\right)=\frac{(1+b) p_{o}-\left(1+p_{o}\right) p_{s}^{A}}{2+p_{o}}$. Substituting the above results into $\Pi_{s}^{A}$ yields:

$$
\begin{aligned}
\Pi_{s}^{A}= & p_{s}^{A} \cdot \frac{\left(1+p_{o}\right)\left(p_{o}+b p_{o}-p_{s}^{A}-p_{o} p_{s}^{A}\right)}{\left(2+p_{o}\right) p_{o}} \\
& +p_{o} \cdot\left(\frac{(1+b) p_{o}+p_{s}^{A}}{\left(2+p_{o}\right) p_{o}}-p_{o}\right) .
\end{aligned}
$$

Then, maximizing the profit of the seller, we can get the optimal agreement price between the seller and the website.

Setting $q_{s}^{A}>0$ yields $p_{s}^{A}>\left(2+p_{o}\right) p_{o}^{2}-(1+b) p_{o}=$ $\frac{1-4 b}{8}$. Solving the first-order and second derivative of $\Pi_{s}^{A}$ with respect to $p_{s}^{A}$ yields:

$$
\begin{aligned}
& \frac{\partial \Pi_{s}^{A}}{\partial p_{s}^{A}}=\frac{\left(1+b-2 p_{s}^{A}\right) p_{o}^{2}+\left(2+b-4 p_{s}^{A}\right) p_{o}-2 p_{s}^{A}}{\left(2+p_{o}\right) p_{o}} \\
& \frac{\partial^{2} \Pi_{s}^{A}}{\partial p_{s}^{A^{2}}}=-\frac{2\left(a+p_{o}\right)^{2}}{\left(2 a+p_{o}\right) p_{o}} \leq 0
\end{aligned}
$$

which means that there is a maximum value of $\Pi_{s}^{A}$ with respect to $p_{s}^{A}$. Solving $\frac{\partial \Pi_{s}^{A}}{\partial p_{s}^{A}}=0$, we get the optimal agreement price $p_{s}^{A^{*}}=\frac{(b+2) p_{o}+(1+b) p_{o}^{2}}{2\left(1+p_{o}\right)^{2}}=\frac{3 b+5}{18}$. Then, substituting $p_{s}^{A^{*}}$ into $p_{g}^{A^{*}}\left(p_{s}\right)$ and $\gamma^{A^{*}}\left(p_{s}^{A}\right)$, we get the optimal group-buying price and effort, $p_{g}^{A^{*}}=\frac{12 b+14}{45}$ and $\gamma^{A^{*}}=\frac{3 b+1}{30}$. Then, the optimal group-buying discount and online and offline demands follow:

$$
\begin{aligned}
& \beta^{A^{*}}=\frac{p_{g}^{A^{*}}}{p_{o}}=\frac{28+24 b}{45}, \quad q_{g}^{A^{*}}=\frac{3 b+1}{10}, \\
& q_{s}^{A^{*}}=\frac{48 b+11}{90} .
\end{aligned}
$$

Substituting the above results into the profit of the seller and the website, we get the optimal profits $\Pi_{s}^{A^{*}}=\frac{9 b^{2}+66 b+16}{180}$ and $\Pi_{g}^{A^{*}}=\frac{(3 b+1)^{2}}{360}$, respectively. The total profit of the seller and the website is $\Pi^{A^{*}}=$ $\frac{33+138 b+27 b^{2}}{360}$.

Proof of Proposition 2: The seller can obtain higher profit only under the $\mathrm{O}+\mathrm{GBW}$ channel, with regard to Agreement Price Contract, than under the offline channel. Thus, we get $\Pi_{s}^{A^{*}}=\frac{9 b^{2}+66 b+16}{180}>\Pi_{o}^{*}=\frac{1}{4}$, and then $b \geq \frac{5 \sqrt{6}-11}{3}$.

Pricing coordination of the seller and the GBW proof of Lemma 3: Solving the optimal groupbuying price and effort regarding GBW's effort, we get $p_{g}^{T^{*}}=\frac{1+b+2 p_{s}^{T S}}{5}$ and $p_{g}^{T^{*}}=\frac{1+b+2 p_{s}^{T S}}{5}$. Then the groupbuying channel and offline channel demands should be 
$q_{g}^{T}=\frac{3+3 b-9 p_{s}^{T S}}{5}$ and $q_{s}^{T}=\frac{4 b-2+4 p_{s}^{T}}{10}$. The seller's profit should be $\Pi_{s}^{T^{*}}=\frac{b+3 b p_{s}^{T}-9\left(p_{g}^{T}\right)^{2}}{5}+p_{s}^{T S}-\frac{1}{20}+F$. Solving the first order derivative of seller's profit with regard to the agreement price and fixed payment, we obtain $\frac{\partial \Pi_{s}^{T^{*}}}{\partial p_{s}^{T}}=\frac{3 b-18 p_{s}^{T}}{5}+1$ and $\frac{\partial \Pi_{s}^{T^{*}}}{\partial F}=1$. Thus, we can get:

$$
\begin{aligned}
& p_{s}^{T^{*}}=\frac{3 b+5}{18}, \quad p_{g}^{T^{*}}=\frac{14+12 b}{45}, \quad \gamma^{T^{*}}=\frac{1+3 b}{30} \\
& q_{g}^{T^{*}}=\frac{1+3 b}{10}, \quad q_{s}^{T^{*}}=\frac{11+48 b}{90}
\end{aligned}
$$

The seller's profit is $\Pi_{s}^{T^{*}}=\frac{b^{2}}{20}+\frac{11 b}{30}+\frac{4}{45}+F$ and the GBW's profit is $\Pi_{g}^{T^{*}}=\frac{1+6 b+9 b^{2}}{360}-F \cdot \frac{\partial \Pi_{s}^{T^{*}}}{\partial T}=1>0$, so, when $\Pi_{g}^{T^{*}}=0, F$ has the maximum $F^{T^{*}}=\frac{1+6 b+9 b^{2}}{360}$. Then, $\Pi_{s}^{T^{*}}=\frac{11+46 b+9 b^{2}}{120}$.

Proof of Proposition 3: Only if the seller obtains more profit under the $\mathrm{O}+\mathrm{GBW}+\mathrm{T}$ model than under O model, will it adopt the group-buying channel. Thus, we get $\Pi_{s}^{T^{*}}=\frac{11+46 b+9 b^{2}}{120}>\Pi_{o}^{*}=\frac{1}{4}$, that is $b \geq$ $\frac{10 \sqrt{7}-23}{9}$.

Proof of Proposition 4: $F^{T^{*}}=\frac{1+6 b+9 b^{2}}{360}$ and $\frac{\partial F^{T^{*}}}{\partial b}=\frac{3 b+1}{60}>0$, so when $b$, increases the seller obtains higher fixed payment.

Proof of Lemma 4: Solving the optimal groupbuying price and group-buying effort with regard to GBW's profit, we get:

$$
p_{g}^{R^{*}}=\frac{(1-l)(b+1)+(2-l) p_{s}^{R}}{(1-l)(l+5)}
$$

and:

$$
\gamma^{R^{*}}=\frac{(1-l)(b+1)-3 p_{s}^{R}}{l+5} .
$$

Then, the profit of the seller is obtained by the equation shown in Box I.
Solving the first order derivative of $\Pi_{s}^{R}$ with respect to $p_{s}^{R}$, we get:

$$
\begin{aligned}
& \frac{\partial \Pi_{s}^{R}}{\partial p_{s}^{R}}= \\
& \frac{25+15 b(1-l)^{2}+9 l^{2}-l^{3}-90 p_{s}^{R}+l\left(-33+36 p_{s}^{R}\right)}{\left(-5+4 l+l^{2}\right)^{2}},
\end{aligned}
$$

and solving the second derivative of $p_{s}^{R}$ with respect to $\Pi_{s}^{R}$, we get:

$$
\frac{\partial^{2} \Pi_{s}^{R}}{\partial p_{s}^{R^{2}}}=\frac{-90+36 l}{\left(-5+4 l+l^{2}\right)^{2}}<0 .
$$

So, $\frac{\partial \Pi_{s}^{R}}{\partial p_{s}^{R}}$ decreases with $p_{s}^{R}$. For $p_{s}^{R}$, one meets the condition $0 \leq p_{s}^{R} \leq \frac{1}{2}$. When $p_{s}^{R}=0$ :

$$
\frac{\partial \Pi_{s}^{R}}{\partial p_{s}^{R}}=\frac{15 b(1-l)^{2}+9 l^{2}-l^{3}-20-15 l}{\left(-5+4 l+l^{2}\right)^{2}}<0,
$$

and when $p_{s}^{R}=\frac{1}{2}$ :

$$
\frac{\partial \Pi_{s}^{R}}{\partial p_{s}^{R}}=\frac{15 b(1-l)^{2}+9 l^{2}-l^{3}-20-15 l}{\left(-5+4 l+l^{2}\right)^{2}}<0 .
$$

So, there exists a $p_{s}^{R^{*}}, 0<p_{s}^{R^{*}}<\frac{1}{2}$, that makes $\frac{\partial \Pi_{s}^{R}}{\partial p_{s}^{R}}=0$. Then, setting $\frac{\partial \Pi_{s}^{R}}{\partial p_{s}^{R}}=0$, we get $p_{s}^{R^{*}}=$ $\frac{25+15 b(1-l)^{2}+9 l^{2}-l^{3}-33 l}{90-36 l}$. substituting $p_{s}^{R^{*}}$ into $\Pi_{s}^{R}$, we get $\Pi_{s}^{R}=\frac{9 b^{2}+b(66-30 l)+(4-l)^{2}}{36(5-2 l)}$. Solving the first derivative of $\Pi_{s}^{R}$ with respect to $l$, we get $\frac{\partial \Pi_{s}^{R}}{\partial l}=$ $\frac{4+9 b-9 b^{2}-5 l+l^{2}}{18(5-2 l)^{2}} . \quad \frac{\partial^{2} \Pi_{s}^{R}}{\partial l^{2}}=\frac{(1-2 b)^{2}}{2(5-2 l)^{3}}>0$ always stands up. So, $\frac{\partial \Pi_{s}^{R}}{\partial l}$ increases with $l$. For $l$, it is satisfying to have $0 \leq l \leq 1$. When $l=1, \frac{\partial \Pi_{s}^{R}}{\partial l}=\frac{b-b^{2}}{18}>0$ and when $l=0, \frac{\partial \Pi_{s}^{R}}{\partial l}=\frac{4+9 b-9 b^{2}}{18 * 25}>0$. So, when $0 \leq l \leq 1$, $\frac{\partial \Pi_{s}^{R}}{\partial l}>0$ always stands up. Thus, $l^{*}=1$. Then, with respect to $p_{s}^{R^{*}}=0$, we will have:

$$
\begin{aligned}
\Pi_{s}^{R} & =p_{g}^{R} \cdot\left(1-3 p_{g}^{R}+b+\gamma^{R}\right)+p_{o}^{*} \cdot\left(2 p_{g}^{R}-\frac{1}{2}\right) \\
& =p_{g}^{R} \cdot\left(1-3 p_{g}^{R}+b\right)+\frac{1}{2}\left(2 p_{g}^{R}-\frac{1}{2}\right) .
\end{aligned}
$$

$$
\begin{aligned}
& \Pi_{s}^{R}= \\
& 12 b^{2}(1-l)^{2} l-l^{4}+4 l^{3}\left(2-p_{s}^{R}\right)-18 l^{2}\left(1-2 p_{s}^{R}\right)+4 b(1-l)^{2}\left(5+7 l+15 p_{s}^{R}\right)+4 l\left(4-33 p_{s}^{R}+18 p_{s}^{R^{2}}\right) \\
& \frac{-5\left(1-20 p_{s}^{R}+36 p_{s}^{R^{2}}\right)}{4(l-1)^{2}(5+l)^{2}}
\end{aligned}
$$


Setting $\frac{\partial \Pi_{s}^{R}}{\partial p_{g}^{R}}=2+b-6 p_{g}^{R}=0$, we get $p_{g}^{R^{*}}=\frac{2+b}{6}$. Then, $q_{g}^{R}=\frac{b}{2}, q_{s}^{R}=\frac{2 b+1}{6}, \Pi_{g}^{R^{*}}=0$ and $\Pi_{s}^{R^{*}}=\frac{b^{2}+4 b+1}{12}$.

Proof of Proposition 5: Only if the seller obtains more profit under the $\mathrm{O}+\mathrm{GBW}+\mathrm{T}$ model than under O model, will it adopt the group-buying channel. Thus, we get $\Pi_{s}^{R^{*}}=\frac{b^{2}+4 b+1}{12}>\Pi_{o}^{*}=\frac{1}{4}$, that is $b \geq \sqrt{6}-2$.

Proof of Corollary 1: Comparing the profit that the seller obtains under the centralized setting and Agreement Price Contract scenario, we get $\Pi_{s}^{A^{*}}=$ $\frac{9 b^{2}+66 b+16}{180}<\Pi^{C^{*}}=\frac{3+8 b+12 b^{2}}{20}$. It is the same for the two-part Tariff Contract and the Revenue Sharing Contract, that we can get $\Pi_{s}^{T^{*}}=\frac{11+46 b+9 b^{2}}{120}<\Pi^{C^{*}}=$ $\frac{3+8 b+12 b^{2}}{20}$ and $\Pi_{s}^{R^{*}}=\frac{b^{2}+4 b+1}{12}<\Pi^{C^{*}}=\frac{3+8 b+12 b^{2}}{20}$. Thus, the seller is less likely to add the GBW channel under the Agreement Price Contract scenario, the Twopart Tariff Contract scenario and the Revenue Sharing Contract scenario.

Proof of Proposition 6: The total profit of the seller and GBW under the two-part tariff contract is less than that under the centralized setting. Besides, the total profit of the seller and GBW under the revenue sharing contract is also less than that under the centralized setting. Thus, both the two-part tariff contract $(\mathrm{O}+\mathrm{GBW}+\mathrm{T})$ and revenue sharing contract $(\mathrm{O}+\mathrm{GBW}+\mathrm{R})$ cannot coordinate the total profit.

Proof of Lemma 5: Solving the optimal groupbuying price and group-buying effort with regard to GBW's profit, we get:

$p_{g}^{B^{*}}=$

$\frac{l-1+b(l-1-2 e(l-1))-2 p_{s}^{B^{*}}-l p_{s}^{B^{*}}+e\left(2-2 l+6 p_{s}^{B^{*}}\right)}{(l-1)(5-12 e+l)}$

and:

$$
\gamma^{R^{*}}=\frac{1+b-l-b l-3 p_{s}^{B^{*}}}{5-12 e+l}
$$

Setting $p_{g}^{B^{*}}=\gamma^{B^{*}}=\frac{2+b}{5}$, we get $e^{*}=\frac{l+3 b l+5}{6(b+2)}$ and $p_{s}^{B^{*}}=\frac{1-l}{3}$. Then, the group-buying channel and offline channel quantities should be $q_{g}^{B^{*}}=\frac{3 b+1}{5}$ and $q_{s}^{B^{*}}=$ $\frac{4 b+3}{10}$, respectively. The seller's profit is $\Pi_{s}^{B^{*}}=\frac{3}{20}+$ $\frac{11 b}{30}+\frac{b l}{30}+\frac{b^{2} l}{10}$. Solving the first order derivative of $\Pi_{s}^{B^{*}}$ with respect to $l, \frac{d \Pi_{s}^{B *}}{d l}=\frac{b}{30}+\frac{b^{2}}{10}>0$, so, $l^{*}=1$. Then, we can get all results in Lemma 5 .

Proof of Proposition 7: Only if the seller obtains more profit under the $\mathrm{O}+\mathrm{GBW}+\mathrm{RC}$ model than under O model, will it adopt the group-buying channel. Thus, we get $\Pi_{s}^{R C^{*}}=\frac{3+8 b+2 b^{2}}{20}>\Pi_{o}^{*}=\frac{1}{4}$, that is $b \geq \frac{\sqrt{10}-2}{6}$.

Proof of Proposition 8: Comparing the profit of the seller and GBW, with the total profit of the seller and GBW, we can get:

$$
\begin{aligned}
\Pi_{s}^{R^{*}} & =\frac{b^{2}+4 b+1}{12}<\Pi_{s}^{T^{*}}=\frac{11+46 b+9 b^{2}}{120}<\Pi_{s}^{A^{*}} \\
& =\frac{9 b^{2}+66 b+16}{180}<\Pi_{s}^{R C^{*}} \\
& =\frac{3+8 b+2 b^{2}}{20}, \\
\Pi_{g}^{A^{*}} & =\frac{(3 b+1)^{2}}{360}>\Pi_{g}^{R C^{*}}=\Pi_{g}^{R^{*}}=\Pi_{g}^{T^{*}}=0, \\
\Pi^{R^{*}} & =\frac{b^{2}+4 b+1}{12}<\Pi^{T^{*}}=\frac{11+46 b+9 b^{2}}{120}<\Pi^{A^{*}} \\
& =\frac{33+138 b+27 b^{2}}{360}<\Pi^{R C^{*}} \\
& =\frac{3+8 b+2 b^{2}}{20} .
\end{aligned}
$$

Proof of Corollary 2: Solving the first derivative order of the group-buying effort regarding the website scale, we get $\frac{\partial \gamma^{A}}{\partial b}>0, \frac{\partial \gamma^{T}}{\partial b}>0, \frac{\partial \gamma^{R}}{\partial b}>0$, and $\frac{\partial \gamma^{R C}}{\partial b}>0$, which means the GBW will make more effort to attract new consumers.

Proof of Corollary 3: Solving the first derivative order of the group-buying effort regarding the website scale, we get $\frac{\partial \Pi_{s}^{A}}{\partial b}>0, \frac{\partial \Pi_{s}^{T}}{\partial b}>0, \frac{\partial \Pi_{s}^{R}}{\partial b}>0, \frac{\partial \Pi_{s}^{R C}}{\partial b}>0$, $\frac{\partial \Pi_{g}^{A}}{\partial b}>0, \frac{\partial \Pi_{g}^{T}}{\partial b}>0, \frac{\partial \Pi_{g}^{R}}{\partial b}>0$, and $\frac{\partial \Pi_{g}^{R C}}{\partial b}>0$.

\section{Appendix B}

\section{Proof of Table 2}

Only Offline Channel: When operating only offline channel, the experienced consumers size is $a$, and the retail price is $p_{o}$. So, the demand should be $q_{o}=a-p_{o}$. Product or service cost is $c$, then, the profit of the seller should be $\Pi_{o}=\left(p_{o}-c\right)\left(a-p_{o}\right)$. Solving the first-order derivative yields $\frac{\partial \Pi_{o}}{\partial p_{o}}=a-2 p_{o}+c$. Maximizing $\Pi_{o}$ with respect to $p_{o}$ yields the equilibrium retail price $p_{o}^{*}=\frac{a+c}{2}$. Then, the optimal profit of the seller should be $\Pi_{o}^{*}=\frac{(a-c)^{2}}{4}$.

\section{Operating group-buying}

Proof of centralized setting: When operating group-buying, the group-buying and the offline de- 
mand, respectively, are:

$$
q_{g}^{C}= \begin{cases}\left(1-\frac{p_{g}^{C}}{p_{o}}\right) a+b+\gamma^{C}-p_{g}^{C}, & p_{g}^{C}<p_{o} \\ 0, & p_{g}^{C} \geq p_{o}\end{cases}
$$

and:

$$
q_{s}^{C}=\left\{\begin{array}{ll}
\frac{p_{g}^{C}}{p_{o}} a-p_{o}, & p_{g}^{C}<p_{o} \\
0, & p_{g}^{C} \geq p_{o}
\end{array} .\right.
$$

Setting $q_{g}^{C} \geq 0$, we get $p_{g}^{C} \leq \frac{\left(a+b+\gamma^{C}\right) p_{o}}{a+p_{o}} \leq \frac{(a+b) p_{o}}{a+p_{o}}=$ $\frac{(a+b)(a+c)}{3 a+c}$; setting $q_{g}^{C} \geq 0$, we get $p_{g}^{C} \geq \frac{p_{o}^{2}}{a}=\frac{(a+c)^{2}}{4 a}$, which also satisfies the condition for $c \leq p_{g}^{C} \leq p_{o}$, that is, $c \leq p_{g}^{C} \leq \frac{a+c}{2}$, under group-buying.

Case 1: In this case, $4 a c+c^{2}-a^{2} \geq 0$ with respect to $c \leq p_{g}^{C} \leq p_{o}$ and $\frac{(a+c)^{2}}{4 a} \leq \frac{a+c}{2} \quad(c \leq a$ always stands up). Here, we can discuss in four kinds of circumstances:

1. $c \leq \frac{(a+c)^{2}}{4 a} \leq \frac{(a+b)(a+c)}{3 a+c} \leq \frac{a+c}{2}$, that is:

$$
\begin{gathered}
\frac{4 a c+c^{2}-a^{2}}{4 a} \leq b \leq \frac{a+c}{2} \\
\Pi_{s}^{C}=\left(p_{g}^{C}-c\right)\left[\left(1-\frac{p_{g}^{C}}{p_{o}}\right) a+b+\gamma^{C}-p_{g}^{C}\right] \\
+\left(p_{o}-c\right)\left(\frac{p_{g}^{C}}{p_{o}} a-p_{o}\right)-\frac{\gamma^{2}}{2} ;
\end{gathered}
$$

2. $c \leq \frac{(a+c)^{2}}{4 a} \leq \frac{a+c}{2} \leq \frac{(a+b)(a+c)}{3 a+c}$, that is:

$$
\begin{aligned}
b \leq & \frac{a+c}{2}, \\
\Pi_{s}^{C}= & \left(p_{g}^{C}-c\right)\left[\left(1-\frac{p_{g}^{C}}{p_{o}}\right) a+b+\gamma^{C}-p_{g}^{C}\right] \\
& +\left(p_{o}-c\right)\left(\frac{p_{g}^{C}}{p_{o}} a-p_{o}\right)-\frac{\gamma^{C^{2}}}{2} ;
\end{aligned}
$$

3. $c \leq \frac{(a+b)(a+c)}{3 a+c} \leq \frac{(a+c)^{2}}{4 a} \leq \frac{a+c}{2}$, that is:

$$
b \leq \frac{4 a c+c^{2}-a^{2}}{4 a}, \quad \Pi_{s}^{C}=\left(p_{o}-C\right)\left(\frac{p_{g}^{C}}{p_{o}} a-p_{o}\right) ;
$$

4. $\frac{(a+b)(a+c)}{3 a+c} \leq c \leq \frac{(a+c)^{2}}{4 a} \leq \frac{a+c}{2}$, that is:

$$
\begin{aligned}
b & \leq \min \left\{\frac{4 a c+c^{2}-a^{2}}{4 a}, \frac{(3 a+c) c}{a+c}-a\right\} \\
& =\frac{4 a c+c^{2}-a^{2}}{4 a} \\
\Pi_{s}^{C} & =\left(p_{o}-C\right)\left(\frac{p_{g}^{C}}{p_{o}} a-p_{o}\right) .
\end{aligned}
$$

In conclusion, when $4 a c+c^{2}-a^{2} \geq 0$, the profit of the seller should be formulated as follows:

$\Pi_{s}^{C}=$

$$
\left\{\begin{aligned}
\left(p_{g}^{c}-c\right)\left[\left(1-\frac{p_{g}^{C}}{p_{o}}\right) a+b+\gamma^{C}-p_{g}^{C}\right] & \\
\quad+\left(p_{o}-c\right)\left(\frac{p_{g}^{C}}{p_{o}} a-p_{o}\right)-\frac{\gamma^{C^{2}}}{2}, & b \geq \frac{4 a c+c^{2}-a^{2}}{4 a} \\
\left(p_{o}-c\right)\left(\frac{p_{g}^{C}}{p_{o}} a-p_{o}\right), & b<\frac{4 a c+c^{2}-a^{2}}{4 a}
\end{aligned}\right.
$$

Sub-case 1: In this sub-case we have: $b \geq \frac{4 a c+c^{2}-a^{2}}{4 a}$. Total profit in centralized setting is:

$$
\begin{aligned}
& \Pi_{s}^{C}=\left(p_{g}^{C}-c\right)\left[\left(1-\frac{p_{g}^{C}}{p_{o}}\right) a+b+\gamma^{C}-p_{g}^{C}\right] \\
& +\left(p_{o}-C\right)\left(\frac{p_{g}^{C}}{p_{o}} a-p_{o}\right)-\frac{\gamma^{C^{2}}}{2}, \\
& \frac{\partial \Pi_{s}^{C}}{\partial p_{g}^{C}}=\frac{\left(2 a+b+c+\gamma^{C}\right) p_{o}-2\left(a+p_{o}\right) p_{g}^{C}}{p_{o}}, \\
& \frac{\partial \Pi_{s}^{C}}{\partial \gamma^{C}}=p_{g}^{C}-C-\gamma^{C}, \quad \frac{\partial^{2} \Pi_{s}^{C}}{\partial p_{g}^{C}}=-2\left(\frac{a}{p_{o}}+1\right), \\
& \frac{\partial^{2} \Pi_{s}^{C}}{\partial \gamma^{C^{2}}}=-1, \quad \frac{\partial^{2} \Pi_{s}^{C}}{\partial p_{g}^{C} \partial \gamma^{C}}=1, \\
& \frac{\partial^{2} \Pi_{s}^{C}}{\partial \gamma^{C} \partial p_{g}^{C}}=1 .
\end{aligned}
$$

Then the Hesse Matrix of $\Pi_{s}$ with respect to $p_{g}^{C}$ and $\gamma^{C}$ is:

$$
H\left(\Pi_{s}^{C}\right)=\left[\begin{array}{cc}
-2\left(\frac{a}{p_{o}}+1\right) & 1 \\
1 & -1
\end{array}\right] .
$$

So, $\Pi_{s}^{C}$ has a maximum value about $p_{g}^{C}$ and $\gamma^{C}$. Setting:

$$
\frac{\partial \Pi_{s}^{C}}{\partial p_{g}^{C}}=\frac{\left(2 a+b+c+r^{C}\right) p_{o}-2\left(a+p_{o}\right) p_{s}^{C}}{p_{o}}=0
$$

and:

$$
\frac{\partial \Pi_{s}^{C}}{\partial \gamma^{C}}=p_{g}^{C}-c-\gamma^{C}=0
$$

yields:

$$
p_{g}^{C^{*}}=\frac{2 a p_{o}+b p_{o}}{2 a+p_{o}}=\frac{(a+c)(2 a+b)}{5 a+c}
$$


and:

$$
\begin{aligned}
\gamma^{C^{*}} & =\frac{(2 a+b-c) p_{o}-2 a c}{2 a+p_{o}} \\
& =\frac{2 a^{2}-3 a c+a b-c^{2}+b c}{5 a+c} .
\end{aligned}
$$

According to $\gamma^{C^{*}} \geq 0$, we get $b \geq \frac{3 a c-2 a^{2}+c^{2}}{a+c}$. Thus, the optimal discount should be $\beta^{C^{*}}=\frac{2(2 a+b)}{5 a+c}$. According to $\beta^{C^{*}} \leq 1$, we get $b \leq \frac{a+c}{2}$. Then, we get:

$$
\begin{aligned}
q_{g}^{C^{*}} & =\frac{(a+b-c) p_{o}+a(b-2 c)}{2 a+p_{o}} \\
& =\frac{a^{2}-4 a c+3 a b-c^{2}+b c}{5 a+c}
\end{aligned}
$$

and:

$$
q_{s}^{C^{*}}=\frac{a\left(2 a p_{o}+b p_{o}\right)}{p_{o}\left(2 a+p_{o}\right)}-p_{o}=\frac{3 a^{2}-6 a c+4 a b-c^{2}}{2(5 a+c)}
$$

As it satisfies $q_{g}^{C^{*}} \geq 0$, we can get $b \geq \frac{4 a c-a^{2}+c^{2}}{3 a+c}$. Then, according to $q_{s}^{C^{*}} \geq 0$, we get $b \geq \frac{6 a c-3 a^{2}+c^{2}}{4 a}$. Hence, we have the relations shown in Box II.

Besides, only when $\Pi_{s}^{C^{*}} \geq \Pi_{o}^{*}$, will the seller cooperate with the website. Thus, when $b \geq$ $\frac{3 a c-2 a^{2}+c^{2}+\left(5 a^{2}-c^{2}-4 a c\right) \sqrt{a /(5 a+c)}}{a+c}$, the seller chooses to provide group-buying.

Sub-case 2: When $b<\frac{4 a c+c^{2}-a^{2}}{4 a}, \Pi_{s}^{C}$ increases with respective to $p_{g}^{C}$. Besides, $p_{g}^{C} \leq p_{o}$, so $p_{g}^{C^{*}}=p_{o}$. Thus $\Pi_{s}^{C^{*}}=\frac{(a-c)^{2}}{4}$, which means seller does not run groupbuying.

Case 2: When $4 a c+c^{2}-a^{2}<0$, there is only one scenario, that is:

$$
\begin{aligned}
\Pi_{s}^{C}= & \left(p_{g}^{C}-c\right)\left[\left(1-\frac{p_{g}^{C}}{p_{o}}\right) a+b+\gamma^{C}-p_{g}^{C}\right] \\
& +\left(p_{o}-c\right)\left(\frac{p_{g}^{C}}{p_{o}} a-p_{o}\right)-\frac{\gamma^{C^{2}}}{2}, \quad b \geq 0 .
\end{aligned}
$$

So, we can get the same optimal result as in Sub-case 1 as shown in Box III.

\section{Proof of $O+G B W+A$ model (agreement price contract)}

The group-buying and offline demand, respectively, are:

$$
q_{g}^{A}= \begin{cases}\left(1-\frac{p_{g}^{A}}{p_{o}}\right) a+b+\gamma^{A}-p_{g}^{A}, & p_{g}^{A}<p_{o} \\ 0, & p_{g}^{A} \geq p_{o}\end{cases}
$$

and:

$$
q_{s}^{A}= \begin{cases}\frac{p_{g}^{A}}{p_{o}} \cdot a-p_{o}, & p_{g}^{A}<p_{o} \\ 0, & p_{g}^{A} \geq p_{o}\end{cases}
$$

According to $q_{g}^{A} \geq 0$, we can get $p_{s}^{A} \leq p_{g}^{A} \leq \frac{a+b+\gamma^{s}}{1+\frac{a}{p_{o}}} \leq$ $\frac{a+b}{1+\frac{a}{p_{o}}} \leq \frac{(a+b) p_{o}}{a+p_{o}}=\frac{(a+b)(a+c)}{3 a+c}$.

Thus, when only $p_{s}^{A} \leq \frac{(a+b)(a+c)}{3 a+c}$, will seller cooperate with the website. Then, according to $q_{s}^{A}\left(p_{s}^{A}\right)=\frac{(a+b) p_{o}+a p_{s}^{A}}{2 a+p_{o}} \cdot \frac{a}{p_{o}}-p_{o} \geq 0$, we get $p_{s}^{A} \geq$ $\frac{p_{o}^{2}\left(2 a+p_{o}\right)}{a^{2}}-\frac{(a+b) p_{o}}{a}=\frac{(a+c)\left(a^{2}+c^{2}+6 a c-4 a b\right)}{8 a^{2}}$.

We firstly maximize $\Pi_{g}^{A}$ with respect to $q_{s}^{A}$ and $\gamma^{A}$. When $q_{g}^{A} \geq 0$ :

$$
\begin{aligned}
& \Pi_{g}^{A}=\left(p_{g}^{A}-p_{s}^{A}\right) \cdot q_{g}^{A}-\frac{\left(\gamma^{A}\right)^{2}}{2} \\
& \frac{\partial \Pi_{g}^{A}}{\partial p_{g}^{A}}=a+b+\gamma^{A}+\left(\frac{a}{p_{o}}+1\right) p_{s}^{A}-2\left(\frac{a}{p_{o}}+1\right) p_{g}^{A}
\end{aligned}
$$

$$
\Pi_{s}^{C^{*}}=\frac{3 a^{3}+8 a^{2} b-13 a^{2} c+12 a b^{2}-12 a b c+11 a c^{2}+2 b^{2} c-4 b c^{2}+3 c^{3}}{4(5 a+c)}, \quad b \geq \frac{4 a c+c^{2}-a^{2}}{4 a}
$$

Box II

$$
\begin{aligned}
& \Pi_{s}^{C^{*}}=\frac{3 a^{3}+8 a^{2} b-13 a^{2} c+12 a b^{2}-12 a b c+11 a c^{2}+2 a b^{2} c-4 b c^{2}+3 c^{3}}{4(5 a+c)}, \\
& b \geq \frac{3 a c-2 a^{2}+c^{2}+\left(5 a^{2}-c^{2}-4 a c\right) \sqrt{a /(5 a+c)}}{a+c}
\end{aligned}
$$




$$
\begin{aligned}
& \frac{\partial \Pi_{g}^{A}}{\partial \gamma^{A}}=p_{g}^{A}-p_{s}^{A}-\gamma^{A}, \quad \frac{\partial^{2} \Pi_{g}^{A}}{\partial p_{g}^{A} \partial \gamma^{A}}=1, \\
& \frac{\partial^{2} \Pi_{g}^{A}}{\partial \gamma^{A} \partial p_{g}^{A}}=1, \quad \frac{\partial^{2} \Pi_{g}^{A}}{\partial p_{g}^{A}}=-2\left(\frac{a}{p_{o}}+1\right), \\
& \frac{\partial^{2} \Pi_{g}^{s}}{\partial \gamma^{A^{2}}}=-1 .
\end{aligned}
$$

Thus, the Hesse Matrix of $\Pi_{g}^{A}$ with respect to $p_{g}^{A}$ and $\gamma^{A}$ is $H\left(\Pi_{g}^{A}\right)=\left[\begin{array}{cc}-2\left(\frac{a}{p_{o}}+1\right) & 1 \\ 1 & -1\end{array}\right]$. Therefore, $\Pi_{g}^{A}$ has a maximum value about $p_{g}^{A}$ and $\gamma^{A}$.

Setting $\frac{\partial \Pi_{g}^{s}}{\partial p_{g}^{A}}=0$ and $\frac{\partial \Pi_{g}^{s}}{\partial \gamma^{A}}=0$, we get $p_{g}^{A^{*}}\left(p_{s}^{A}\right)=$ $\frac{(a+b) p_{o}+a p_{s}^{A}}{2 a+p_{o}}$ and $\gamma^{A^{*}}\left(p_{s}^{A}\right)=\frac{(a+b) p_{o}-\left(a+p_{o}\right) p_{s}^{s}}{2 a+p_{o}}$. Thus, $q_{g}^{A}\left(p_{s}^{A}\right)=\frac{\left(a+p_{o}\right)\left(a p_{o}+b p_{o}-a p_{s}^{A}-p_{o} p_{s}^{A}\right)}{\left(2 a+p_{o}\right) p_{o}}, q_{s}^{A}\left(p_{s}^{A}\right)=\frac{(a+b) p_{o}+a p_{s}^{s}}{2 a+p_{o}}$. $\frac{a}{p_{o}}-p_{o}$, and $\Pi_{s}^{A}=\left(p_{s}^{A}-c\right) \cdot \frac{\left(a+p_{o}\right)\left(a p_{o}+b p_{o}-a p_{s}^{A}-p_{o} p_{s}^{A}\right)}{\left(2 a+p_{o}\right) p_{o}}+$ $\left(p_{o}-c\right) \cdot\left[\frac{(a+b) p_{o}+a p_{s}^{A}}{2 a+p_{o}} \cdot \frac{a}{p_{o}}-p_{o}\right]$.

Case 1: When $-a^{2}+c^{2}+4 a c \geq 0$ :

$\Pi_{s}^{A}=$

$$
\begin{cases}\left(p_{s}^{A}-c\right) \cdot \frac{\left(a+p_{o}\right)\left(a p_{o}+b p_{o}-a p_{s}^{A}-p_{o} p_{s}^{A}\right)}{\left(2 a+p_{o}\right) p_{o}} & \\ +\left(p_{o}-c\right) \cdot\left[\frac{(a+b) p_{o}+a p_{s}^{A}}{2 a+p_{o}} \cdot \frac{a}{p_{o}}-p_{o}\right], & b \geq \frac{4 a c+c^{2}-a^{2}}{4 a} \\ \left(p_{o}+c\right)\left(\frac{p_{g}^{A}}{p_{o}} a-p_{o}\right), & b<\frac{4 a c+c^{2}-a^{2}}{4 a}\end{cases}
$$

Sub-case 1: When $b \geq \frac{-a^{2}+c^{2}+4 a c}{4 a}$ :

$$
\begin{aligned}
& \frac{(a+c)\left(a^{2}+c^{2}+6 a c-4 a b\right)}{8 a^{2}} \leq \frac{(a+b)(a+c)}{3 a+c}, \frac{\partial \Pi_{s}^{A}}{\partial p_{s}^{A}} \\
& =\frac{\left(a+b+c-2 p_{s}^{A}\right) p_{o}^{2}+\left(2 a^{2}+a b+2 a c-4 a p_{s}^{A}\right) p_{o}-2 a^{2} p_{s}^{A}}{\left(2 a+p_{o}\right) p_{o}}
\end{aligned}
$$

and:

$$
\frac{\partial^{2} \Pi-s^{A}}{\partial p_{s}^{A^{2}}}=-\frac{2\left(a+p_{o}\right)^{2}}{\left(2 a+p_{o}\right) p_{o}} \leq 0 .
$$

Thus $\Pi_{s}^{A}$ has a maximum value with respect to $p_{s}^{A}$. Setting $\frac{\partial \Pi_{s}^{A}}{\partial p_{s}^{A}}=0$, we get:

$$
\begin{aligned}
p_{s}^{A^{*}} & =\frac{\left(a b+2 a c+2 a^{2}\right) p_{o}+(a+b+c) p_{o}^{2}}{2\left(a+p_{o}\right)^{2}} \\
& =\frac{(a+c)\left(3 a b+6 a c+b c+5 a^{2}+c^{2}\right)}{2(3 a+c)^{2}} .
\end{aligned}
$$

Substituting $p_{s}^{A^{*}}$ and $p_{o}$ into $p_{g}^{A^{*}}\left(p_{s}^{A}\right)$ and $\gamma^{A^{*}}\left(p_{s}^{A}\right)$, we get:

$p_{g}^{A^{*}}=$

$\frac{(a+c)\left(12 a^{2} b+2 a c^{2}+12 a^{2} c+12 a c^{2}+b c^{2}+14 a^{3}+7 a b c\right)}{(3 a+c)^{2}(5 a+c)}$

and:

$$
\gamma^{A^{*}}=\frac{(a+c)\left(3 a b-4 a c+b c+a^{2}-c^{2}\right)}{2(3 a+c)(5 a+c)} .
$$

Then, the optimal group-buying discount should be:

$$
\beta^{A^{*}}=\frac{28 a^{3}+24 a^{2} c+24 a^{2} b+4 a c^{2}+14 a b c+2 b c^{2}}{(3 a+c)^{2}(5 a+c)} .
$$

The group-buying and the offline demand, respectively, are:

$$
q_{g}^{A^{*}}=\frac{b(3 a+c)-4 a c+a^{2}-c^{2}}{2(5 a+c)},
$$

and:

$$
\begin{aligned}
& q_{s}^{A^{*}}= \\
& \frac{4 a b(4 a+c)(3 a+c)+\left(-4 a c+a^{2}-c^{2}\right)\left(8 a c+11 a^{2}+c^{2}\right)}{2(5 a+c)(3 a+c)^{2}} .
\end{aligned}
$$

When running group-buying, it should satisfy $q_{g}^{A^{*}} \geq 0$ and $q_{s}^{A^{*}} \geq 0$. Thus, we get $b \geq \frac{-a^{2}+4 a c+c^{2}}{3 a+c}$. The optimal profit of the seller and the website, respectively, are obtained by the equations shown in Box IV.

$$
\begin{aligned}
& 16 a^{5}+66 a^{4} b-74 a^{4} c+9 a^{3} b^{2}-44 a^{3} b c+12 a^{3} c^{2}+15 a^{2} b^{2} c-64 a^{2} b c^{2}+56 a^{2} c^{3}+7 a b^{2} c^{2}-20 a b c^{3} \\
& \Pi_{s}^{A^{*}}=\frac{+20 a c^{4}+b^{2} c^{3}-2 b c^{4}+2 c^{5}}{4(3 a+c)^{2}(5 a+c)}
\end{aligned}
$$

and:

$$
\Pi_{g}^{A^{*}}=\frac{(a+c)\left(3 a b+b c-4 a c+a^{2}-c^{2}\right)^{2}}{8(5 a+c)(3 a+c)^{2}} .
$$


For the seller, when only $\Pi_{s}^{A^{*}} \geq \Pi_{o}^{*}$, will it cooperate with the website. Thus, when $b \geq$ $\frac{7 a c^{2}+11 a^{2} c-11 a^{3}+c^{3}+(a-c)(5 a+c) \sqrt{2 a(3 a+c)}}{(3 a+c)(a+c)}$, the seller and the website will cooperate to provide group-buying.

Sub-case 2: $b<\frac{-a^{2}+c^{2}+4 a c}{4 a}$, that is $\frac{(a+c)\left(a^{2}+c^{2}+6 a c-4 a b\right)}{8 a^{2}}$ $>\frac{(a+b)(a+c)}{3 a+c}$.

$3 a+c$
In this scenario, the seller only runs the offline retail. Thus, the optimal profit of the seller should be $\Pi_{s}^{A^{*}}=\frac{(a-c)^{2}}{4}$.

In conclusion, only when:

$b \geq$

$\frac{7 a c^{2}+11 a^{2} c-11 a^{3}+c^{3}+(a-c)(5 a+c) \sqrt{2 a(3 a+c)}}{(3 a+c)(a+c)}$,

the seller and the website cooperate with each other.

Case 2: $-a^{2}+c^{2}+4 a c<0$. In this case, there is just one scenario that is:

$$
\begin{aligned}
\Pi_{s}^{A} & =\left(p_{s}^{A}-c\right) \cdot\left[\left(1-\frac{p_{g}^{A}}{p_{o}}\right) a+b+\gamma^{A}-p_{g}^{A}\right] \\
& +\left(p_{o}-c\right) \cdot\left(\frac{p_{g}^{A}}{p_{o}} \cdot a-p_{o}\right), \quad b \geq 0 .
\end{aligned}
$$

Thus, we get the same optimal results as in Case 1. Only when:

$$
\begin{aligned}
& b \geq \\
& \frac{7 a c^{2}+11 a^{2} c-11 a^{3}+c^{3}+(a-c)(5 a+c) \sqrt{2 a(3 a+c)}}{(3 a+c)(a+c)},
\end{aligned}
$$

the seller and the website cooperate with each other.

Form Case 1 and Case 2, it is concluded that when $b \geq \frac{7 a c^{2}+11 a^{2} c-11 a^{3}+c^{3}+(a-c)(5 a+c) \sqrt{2 a(3 a+c)}}{(3 a+c)(a+c)}$, the seller and the website cooperate with each other to provide group-buying; otherwise, the seller only run the offline retail.

\section{Proof of $O+G B W+R C$ model (revenue-cost sharing contract)}

Solving the optimal group-buying price and groupbuying effort with regard to GBW's profit, we get the equations shown in Box V. Setting $p_{g}^{B^{*}}=$ $\frac{(2 a+b)(a+c)}{5 a+c}$ and $\gamma^{B^{*}}=\frac{2 a^{2}+a b-3 a c+b c-c^{2}}{2(2 a+b)}$, we get $e^{*}=\frac{a^{3} l-a c^{2}+4 a^{2}-c^{3} l+5 a^{3}+3 a^{2} b l-5 a c^{2} l-3 a^{2} c l+b c^{2} l+4 a b c l}{2(3 a+c)\left(2 a^{2}-3 a c+a b-c^{2}+b c\right)}$ and $p_{s}^{B^{*}}=\frac{(a+c)^{2}(1-l)}{(3 a+c)}$. Thus, the group-buying channel and offline channel quantities should be $q_{g}^{B^{*}}=\frac{6 a^{3}+a^{2}(11 b-17 c)+(b-c) c^{2}+4 a\left(b^{2}+b c-2 c^{2}\right)}{2(2 a+b)(5 a+c)}$ and $q_{s}^{B^{*}}=$ $\frac{3 a^{2}+4 a b-6 a c-c^{2}}{2(5 a+c)}$, respectively. Seller's profit is obtained by the equation shown in Box VI.

Solving $\Pi_{s}^{B^{*}}$ 's first order derivative with respect to $l$, we get $\frac{d \Pi_{s}^{B}}{d l}=\frac{(b-c)(a+c)\left(a^{2}+3 a b-4 a c+b c-c^{2}\right)}{30 a^{2}+16 a c+2 c^{2}}>0$. Thus, we get $l^{*}=1, p_{s}^{B^{*}}=0$, and $e^{*}=\frac{1}{2}$.

The seller and GBW's profit are, respectively, obtained by the equations shown in Box VII, and the total profit is obtained by the equation shown in Box VIII. Only if:

$$
b \geq \frac{3 a c-2 a^{2}+c^{2}+\left(5 a^{2}-c^{2}-4 a c\right) \sqrt{a /(5 a+c)}}{a+c},
$$

the seller will choose the group-buying channel.

Only under this scenario, will the seller and the website cooperate with each other. Otherwise, they will not cooperate.

$$
p_{g}^{B^{*}}=\frac{\left(a^{2}(l-1-2 e(l-1))-a\left(b(2 e-1)(l-1)+c(2 e-1)(l-1)+(2-6 e+l) p_{s}^{B}\right)-c\left(b(2 e-1)(l-1)+(l-2 e) p_{s}^{B}\right)\right)}{(l-1)(a(5-12 e+l)+c(1-4 e+l))}
$$

and:

$$
\gamma^{B^{*}}=\frac{a^{2}(1-l)+c\left(b(1-l)-p_{s}^{B}\right)+a\left(b(1-l)+c(1-l)-3 p_{s}^{B}\right)}{c(1-4 e+l)+a(5-12 e+l)} .
$$

Box V

$$
\begin{aligned}
& 9 a^{4}+2 a^{3}(b(11+l)-c(17+l))+c^{2}\left(2 b^{2} l-4 b c l+c^{2}(1+2 l)\right)+2 a^{2}\left(3 b^{2} l-2 b c(4+3 l)+c^{2}(7+3 l)\right) \\
& \Pi_{s}^{B^{*}}=+2 a c\left(4 b^{2} l+5 c^{2}(1+l)-3 b(c+3 c l)\right) \\
& 4\left(15 a^{2}+8 a c+c^{2}\right)
\end{aligned}
$$




$$
\begin{aligned}
& \Pi_{s}^{B^{*}}=\frac{3 a^{3}+8 a^{2} b+2 a b^{2}-13 a^{2} c-12 a b c+2 b^{2} c+11 a c^{2}-4 b c^{2}+3 c^{3}}{4(5 a+c)} \\
& \Pi_{g}^{B^{*}}=0 .
\end{aligned}
$$

Box VII

$$
\Pi^{B^{*}}=\frac{3 a^{3}+8 a^{2} b+2 a b^{2}-13 a^{2} c-12 a b c+2 b^{2} c+11 a c^{2}-4 b c^{2}+3 c^{3}}{4(5 a+c)} .
$$

\section{Box VIII}

\section{Proof of Proposition 9:}

1. Solving the first order derivative of group-buying price with respect to the cost, we get $\frac{\partial p_{g}^{A^{*}}}{\partial c}>0$, $\frac{\partial p_{g}^{T^{*}}}{\partial c}>0, \frac{\partial p_{g}^{R^{*}}}{\partial c}>0$, and $\frac{\partial p_{g}^{R C^{*}}}{\partial c}>0$, and solving the first order derivative of the agreement price with respect to the cost we get $\frac{\partial p_{s}^{A^{*}}}{\partial c}>0, \frac{\partial p_{s}^{T^{*}}}{\partial c}>0$, $\frac{\partial p_{s}^{R^{*}}}{\partial c}>0$, and $\frac{\partial p_{s}^{R C^{*}}}{\partial c}>0$.

2. $\frac{\partial \beta^{A^{*}}}{\partial c}>0, \frac{\partial \beta^{T^{*}}}{\partial c}>0, \frac{\partial \beta^{R^{*}}}{\partial c}>0, \frac{\partial \beta^{R C^{*}}}{\partial c}>0$, thus, when $a$ increases, the group-buying discount is smaller.

3. For the group-buying and offline demand, when the cost increases: $\frac{\partial q_{g}^{A^{*}}}{\partial c}<0, \frac{\partial q_{g}^{T^{*}}}{\partial c}<0, \frac{\partial q_{g}^{R^{*}}}{\partial c}<0$, $\frac{\partial q_{g}^{R C^{*}}}{\partial c}<0, \frac{\partial q_{s}^{A^{*}}}{\partial c}<0, \frac{\partial q_{s}^{T^{*}}}{\partial c}<0, \frac{\partial q_{s}^{R^{*}}}{\partial c}<0$, and $\frac{\partial q_{s}^{R C^{*}}}{\partial c}<0$.

4. For the profit of the seller and the GBW: $\frac{\partial \Pi_{s}^{A^{*}}}{\partial c}<$ $0, \frac{\partial \Pi_{s}^{T^{*}}}{\partial c}<0, \frac{\partial \Pi_{s}^{R^{*}}}{\partial c}<0, \frac{\partial \Pi_{s}^{R C^{*}}}{\partial c}<0, \frac{\partial \Pi_{g}^{A^{*}}}{\partial c}<0$, $\frac{\partial \Pi_{g}^{T^{*}}}{\partial c}<0, \frac{\partial \Pi_{g}^{R^{*}}}{\partial c}<0$ and $\frac{\partial \Pi_{g}^{R C^{*}}}{\partial c}<0$.

\section{Proof of Proposition 10:}

1. Solving the first order derivative of group-buying price with respect to the size of experienced consumers, we get $\frac{\partial p_{g}^{A^{*}}}{\partial a}>0, \frac{\partial p_{g}^{T^{*}}}{\partial a}>0, \frac{\partial p_{g}^{R^{*}}}{\partial a}>0$, and $\frac{\partial p_{g}^{R C^{*}}}{\partial a}>0$, and solving the first order derivative of the agreement price with respect to the size of experienced consumers, we get $\frac{\partial p_{s}^{A^{*}}}{\partial a}>0, \frac{\partial p_{s}^{T^{*}}}{\partial a}>0$, $\frac{\partial p_{s}^{R^{*}}}{\partial a}>0$, and $\frac{\partial p_{s}^{R C^{*}}}{\partial a}>0$.

2. $\frac{\partial \beta^{A^{*}}}{\partial a}>0, \frac{\partial \beta^{T^{*}}}{\partial a}>0, \frac{\partial \beta^{R^{*}}}{\partial a}>0$, and $\frac{\partial \beta^{R C^{*}}}{\partial a}>0$, thus, when $a$ increases, the group-buying discount is smaller.
3. For the group-buying and offline demands, when the cost increases, $\frac{\partial q_{g}^{A^{*}}}{\partial a}<0, \frac{\partial q_{g}^{T^{*}}}{\partial a}<0, \frac{\partial q_{g}^{R^{*}}}{\partial a}<0$, $\frac{\partial q_{g}^{R C^{*}}}{\partial a}<0, \frac{\partial q_{s}^{A^{*}}}{\partial a}<0, \frac{\partial q_{s}^{T^{*}}}{\partial a}<0, \frac{\partial q_{s}^{R^{*}}}{\partial a}<0$, and $\frac{\partial q_{s}^{R C^{*}}}{\partial a}<0$.

4. For the profit of the seller and the GBW: $\frac{\partial \Pi_{s}^{A^{*}}}{\partial a}<$ $0, \frac{\partial \Pi_{s}^{T^{*}}}{\partial a}<0, \frac{\partial \Pi_{s}^{R^{*}}}{\partial a}<0, \frac{\partial \Pi_{s}^{R C^{*}}}{\partial a}<0, \frac{\partial \Pi_{g}^{A^{*}}}{\partial a}<0$, $\frac{\partial \Pi_{g}^{T^{*}}}{\partial a}<0, \frac{\partial \Pi_{g}^{R^{*}}}{\partial a}<0$, and $\frac{\partial \Pi_{g}^{R C^{*}}}{\partial a}<0$.

\section{Biographies}

Yifan Wu is an Associate Professor in School of Business, East China University of Science and Technology. He received his $\mathrm{BE}$ and $\mathrm{PhD}$ in Industrial Engineering and Management from Shanghai Jiao Tong University in 2004 and 2010, respectively. His current research interests include platform business model, sustainable operations, and operations/marketing interfaces.

Danning Li received her MS degree in Management Science and Engineering from East China University of Science and Technology. She currently works as a business and integration arch analyst in Accenture Co., LTD China. Her research interests include supply chain management, group buying, and e-commerce.

Tian Li is an Associate Professor at the School of Business in East China University of Science and Technology. She obtained her PhD in Operations Management from The Hong Kong University of Science and Technology. Her research interests include operationsmarketing interface, supply chain competition, and information sharing and contract design in supply chains. 